Nodel ing and sem-act i ve fuzzy control of magnet or heol ogi cal el ast omer-based i sol at or for sei smic response reduct i on

\begin{tabular}{|l|l|}
\hline 著者 & $\begin{array}{l}\text { Nguyen Xuan Bao, Komat suzaki Toshi hi ko, I wat a } \\
\text { Yoshi o, Asanuma Har uhi ko }\end{array}$ \\
\hline 著者別表示 & 小松崎 俊彦, 岩田 佳雄, 浅沼 春彦 \\
\hline $\begin{array}{l}\text { j ournal or } \\
\text { publ i cat i on titl e }\end{array}$ & Mechani cal Syst ens and Si gnal Processi ng \\
\hline vol une & 101 \\
\hline page range & $449-466$ \\
\hline year & $2018-02-15$ \\
\hline URL & ht t p: //doi . or g/10. 24517/00049676 \\
\hline
\end{tabular}




\title{
Modeling and semi-active fuzzy control of magnetorheological elastomer-based isolator for seismic response reduction
}

\author{
Xuan Bao Nguyen ${ }^{\text {a, }}$, Toshihiko Komatsuzaki ${ }^{\text {b, }}$, Yoshio Iwata ${ }^{\text {b }}$, Haruhiko Asanuma ${ }^{\text {b }}$ \\ a Graduate School of Natural Science and Technology, Kanazawa University, Kakuma-machi, Kanazawa, \\ Ishikawa 920-1192 JAPAN \\ E-mail: xuanbao233@gmail.com \\ ${ }^{\mathrm{b}}$ Institute of Science and Engineering, Kanazawa University \\ E-mail: toshi@se.kanazawa-u.ac.jp, iwata@se.kanazawa-u.ac.jp, h-asanuma@se.kanazawa-u.ac.jp \\ * Corresponding author. Tel.: +81 76234 4675; fax: +81 762344676 \\ E-mail:xuanbao233@gmail.com; toshi@se.kanazawa-u.ac.jp
}

\begin{abstract}
In this paper, a magnetorheological elastomer (MRE) based isolator was investigated to mitigate excessive vibrations in structures during seismic events. The primary objectives of this research are to propose a numerical model that expresses viscoelastic behaviors of the MRE and predict operation process of the MRE-based isolator for future design of isolator systems for various technical applications. Despite the simplicity in parameter definition in comparison to the conventional models, the proposed model works efficiently in a wide range of frequencies and amplitudes. The model consists of the following components: viscoelasticity of host MRE , magnetic field-induced property, nominal viscosity as well as high stiffness in low excitation frequency that are modeled in analogy with a standard linear solid model (Zener model), a stiffness variable spring, and a smooth Coulomb friction, respectively. Furthermore, a semi-active fuzzy controller was designed to enhance the performance of the isolator in suppressing structural vibrations. The control strategy was built to determine the command applied current. The controller is completely adequate for handling the nonlinearity of the isolator and works independently with the building structure. The efficiency of the MRE-based isolator was evaluated by the responses of the scaled building under seismic excitation. Numerical and experimental results show that the isolator accompanied with a fuzzy controller remarkably reduces the relative displacement and absolute acceleration of the scaled building compared to passive-off and passive-on cases.
\end{abstract}

Keywords: Magnetorheological elastomer; Modeling; Fuzzy logic; Semi-active control; Seismic response. 


\section{INTRODUCTION}

Magnetorheological elastomer (MRE) is a class of smart materials that mainly generate a slightly magnetic field dependent variable stiffness. MREs have attracted significant interest for application in the field of intelligent devices, such as vibration absorbers and isolators [1, 2]. Recently, MREs have been used effectively for base isolation of structure to protect structures from seismic vibrations [3, 4]. The MRE-based isolators have the ability to govern the transmissibility by adjusting their properties such as stiffness and damping.

In order to design MRE-based isolator systems for various technical applications, a numerical model is necessary to represent dynamic behaviors of MRE. Unfortunately, these behaviors are strongly nonlinear functions of magnetic flux density and displacement amplitude, and they are also affected by changes in frequencies to some extent [5]. Therefore, modeling of the MRE properties is a substantial challenge, particularly in vibration control technology. Recently, different viewpoints of MRE modeling were considered. Li et al. [6] proposed a micromechanics-based viscoelastic model with chain structure that predicted magnetic-field-dependent dynamic shear stiffness and damping of MRE. Li et al. [7] developed a four-parameter viscoelastic model for MRE. In this model, a spring element is in parallel with the standard Kelvin-Voigt model that predicts the viscoelastic properties of MRE under harmonic loadings. However, the strain amplitude is not above $10 \%$ and frequency is less than $10 \mathrm{~Hz}$. Eem et al. [8] developed a nonlinear dynamic model that combined the Ramber-Osgood model and the Maxwell model. Simple algebraic equations are used to represent hysteretic nonlinearity. However, its parameters are independent of displacement and frequency. The use of Bouc-Wen (1976) model to represent the nonlinear hysteresis is well known in MR fluid model. The Bouc-Wen model is well acceptable in MRE modeling in recent years [9]. However, one of the major problems in the model is the need for identification of its seven parameters. Norouzi et al. [10] proposed a modified Kelvin-Voigt viscoelastic model for MRE-based isolator, whose coefficients are calculated by nonlinear regression technique. This model only works effectively in the low-frequency range.

The MRE-based isolator is one of the semi-active devices that require an efficient controller. Because

of nonlinearity in the model, not many control algorithms exist that could effectively operate MRE devices. The on-off algorithm is widely used [11, 12]. Opie et al. [13] developed a clipped-optimal controller for an MRE-based isolator. Behrooz et al. [14] used Lyapunov algorithm in seismic control. Du et al. [15] applied a sub-optimal H- $\infty$ strategy to suppress the vibration of a vehicle seat suspension. In these algorithms, the command applied current has only two options: either zero or the maximum value. Consequently, fast switching produces periodical acceleration and jerk peaks that result in negative effects on the quality of structures. Choi et al. [16] developed a semi-active fuzzy algorithm for seismic response of three story building using MR damper. The command voltage is completely based on the structural 
first-floor velocity and the third-floor velocity. The semi-active fuzzy algorithm has advantages over the algorithms mentioned above and effectively reduces the building structural responses.

In this study, a MRE-based isolator was investigated to mitigate the seismic performance. The dynamic viscoelastic model of MRE was developed to simulate the dynamic behavior of MRE. The developed model works efficiently in a wide range of frequencies and amplitudes. A procedure was proposed that can determine the parameters in the model. A semi-active fuzzy controller was designed for seismic protection of building with an MRE-based isolator. The applied current is generated according to the relative displacement, the relative velocity, and the ground acceleration. The developed controller is successful in reducing the relative response as well as the absolute acceleration response.

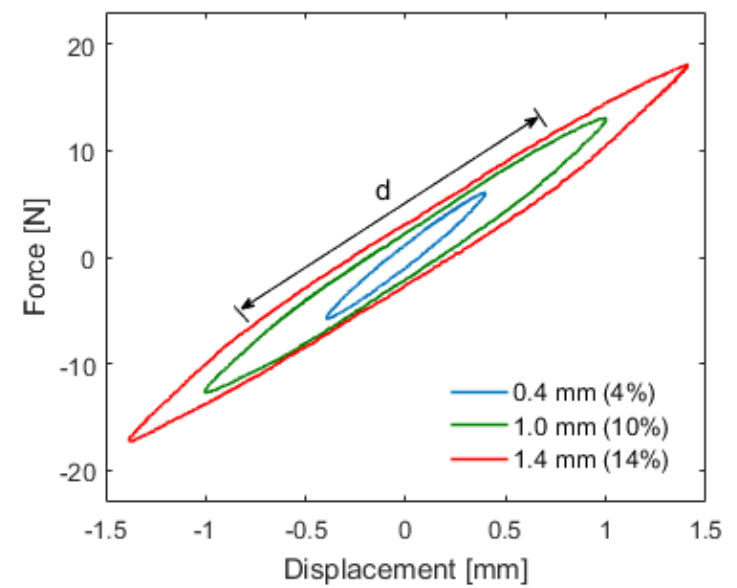

(a)

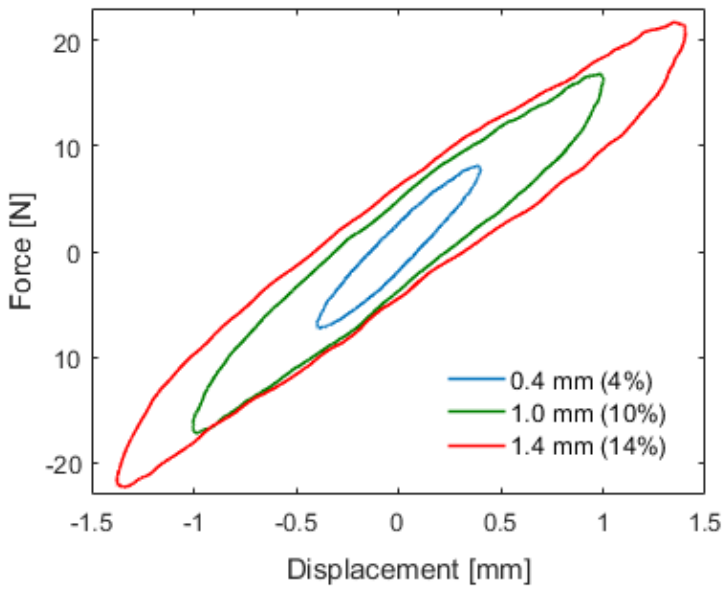

(b)

Fig. 1 Force-displacement response for MRE to harmonic excitations: (a) low frequency (1Hz), (b) medium frequency $(15 \mathrm{~Hz})$.

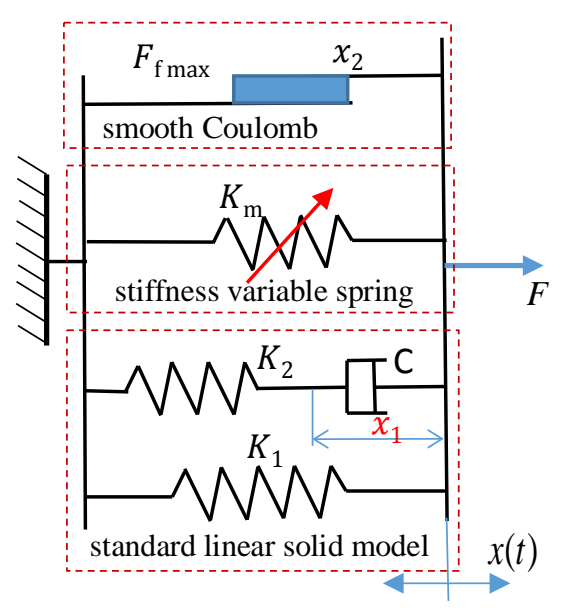

Fig. 2 MRE component model: a standard linear solid model, a stiffness variable spring, and a smooth Coulomb friction connected in parallel. 


\section{DYNAMIC MODEL OF MRE}

\subsection{Properties of MRE}

The elastomer properties of MRE are depicted by force-displacement loops as shown in Fig. 1. Three displacement amplitudes are considered: small amplitude, $0.4 \mathrm{~mm}$; medium amplitude, $1.0 \mathrm{~mm}$; and large amplitude, $1.4 \mathrm{~mm}$. Measurements are performed for two levels of frequency: low frequency, $1 \mathrm{~Hz}$; and medium frequency, $15 \mathrm{~Hz}$. As can be seen in Fig. 1(a), there is an existence of the hysteresis loop at low frequency. The loops maintain their shape if the excitation frequency is relatively low, and therefore present a nominal viscous behavior in MRE. The slope of the hysteresis loops increases as the excitation amplitude decreases, thus the equivalent stiffness increases in small amplitude. The nominal viscosity as well as the increasing stiffness in small amplitude are similar to frictional behaviors. It is shown in Fig. 1(a) that the tangent of the loop for $x \gg-x_{0}$ or $x \ll x_{0}$ (segment $d$ ), where $x_{0}$ denotes the displacement amplitude, approaches a constant value especially in large excitation amplitude. Thus, the nominal stiffness is linear. Figure 1(b) shows that the hysteresis loops become more elliptical with increasing frequency. The increasing stiffness as well as the hysteresis being more elliptical with the increasing frequency are due to the frequency-dependent viscous effect. Therefore, the viscous property of MRE consists of the nominal viscosity and the viscosity affected by frequency. Consequently, the MRE generates a nonlinear viscous behavior besides the linear stiffness. The similar trends are also observed for all values of applied currents. The MRE properties, which depend on excitation frequency, excitation amplitude, and magnetic flux density, are clarified in [5].

\subsection{Dynamic Model of MRE-based isolator}

In order to record the dynamic properties of the MRE deforming in shear direction, a dynamic system was modeled, as shown in Fig. 2. The model consists of a standard linear solid model, a stiffness variable spring, and a smooth Coulomb friction. A standard linear solid model consists of the Maxwell model in parallel with a linear spring, so that the frequency-dependent viscosity and the linear stiffness property of the host MRE can be represented. The relationship between force and displacement can be described as follows:

$$
\begin{gathered}
F_{1}=K_{1} x, \\
F_{2}=C \dot{x}_{1}, \\
F_{2}=K_{2}\left(x-x_{1}\right), \\
F_{v}=F_{1}+F_{2}, \\
\Delta E_{v}=\frac{\pi \omega C}{1+\left(\omega / \omega_{v}\right)^{2}} x_{0}^{2},
\end{gathered}
$$


where $K_{1}$ is the linear spring component of the material, $K_{2}$ is the stiffness coefficient of viscosity, $C$ and $x_{1}$ are the coefficient and displacement of the viscous component, respectively. $F_{1}$ and $F_{2}$ are the elastic force and the viscous force, $F_{v}$ and $x$ correspond to the viscoelastic force and displacement of the component, $\Delta E_{v}$ is the loss energy per cycle caused by the viscoelastic force , $\omega_{v}=K_{2} / C$ is the characteristic frequency, and $\omega$ is the harmonic excitation frequency.

The Eq. (5) is rewritten as follows.

$$
\Delta E_{v}=\frac{\pi C}{1 / \omega+\omega / \omega_{v}^{2}} x_{0}^{2}
$$

According to Cauchy's inequality, the denominator part of Eq. (6) represents the minimum value $2 / \omega_{v}$ at the characteristic frequency $\omega=\omega_{v}=K_{2} / C$. Consequently, the loss energy, $\Delta E_{v}$, reaches the maximum value of $0.5 \pi K_{2} x_{0}^{2}$.

When MRE is exposed to a magnetic field, the embedded ferromagnetic particles are magnetized. The force of variable stiffness generated by the MRE-based isolator with magnetic flux density, $F_{m}$, is expressed as,

$$
\begin{gathered}
F_{m}=K_{m} x, \\
\Delta E_{m}=0,
\end{gathered}
$$

where $K_{m}$ is the variable stiffness, $\Delta E_{m}$ is the loss energy per cycle caused by the force.

The nominal viscosity as well as the increasing stiffness at small amplitude can be expressed by using a friction force. Note, however, that the Coulomb friction function needs to be smoothed to be able to run the simulations. Therefore, the smooth Coulomb friction model [17] is considered as,

$$
\begin{gathered}
F_{f}=F_{f s}+\frac{x-x_{s}}{\left.x_{2}(1-\operatorname{sgn}(\dot{x}) \alpha)+\operatorname{sgn}(\dot{x})\left(x-x_{s}\right)\right)}\left(F_{f \max }-\operatorname{sgn}(\dot{x}) F_{f s}\right), \\
\Delta E_{f}=2 F_{f \max }\left(2 x_{0}-x_{2}(1+\alpha)^{2} \ln \frac{x_{2}(1+\alpha)+2 x_{0}}{x_{2}(1+\alpha)}\right),
\end{gathered}
$$

where $F_{f \max }$ is the maximum friction force, $x_{2}$ is the displacement needed for the friction force to reach $F_{f}=F_{f \max } / 2, \Delta E_{f}$ is the loss energy per cycle caused by the friction, $F_{f s}$ and $x_{s}$ are the friction force and the displacement at static equilibrium, respectively; $\alpha=F_{f s} / F_{f \max }$ is an auxiliary quantity ranging from -1 to 1 , and $\operatorname{sgn}(\dot{x})$ denotes the signum function of the displacement rate. 
Since three forces are generated in the standard linear solid model, in the stiffness variable spring, and in smooth Coulomb friction element, connected in parallel, the total force $F$, the energy $E$, and the loss of energy $\Delta E$ per cycle, can be expressed as follows,

$$
\begin{gathered}
F=F_{v}+F_{m}+F_{f}, \\
\Delta E=\Delta E_{v}+\Delta E_{f} . \\
E=\int_{0}^{x_{0}} F d t .
\end{gathered}
$$

The equivalent stiffness $K$, and the loss factor $L$, are defined by the following equations,

$$
\begin{gathered}
K=\frac{F_{0}}{x_{0}} \\
L=\frac{\Delta E}{E}
\end{gathered}
$$

where $F_{0}$ is the force amplitude and $x_{0}$ is the displacement amplitude.

\subsection{Experimental setup}

Fabricated MRE samples consist of Room Temperature Vulcanization (RTV) silicone rubber (highstrength condensation-cure type, Shin-etsu KE1416), silicone oil, and iron particles (BASF SG-BH) with an average diameter of $20 \mu \mathrm{m}$. The materials were placed in a mixer for the mixture to become homogenous. The mixture was then placed in a copper mold and compressed to remove air bubbles. Finally, the mixture was cured under a magnetic field of $0.5 \mathrm{~T}$ for 24 hours. An anisotropic elastomer sample was formed in square cuboids of sides $25 \mathrm{~mm}$, thickness $10 \mathrm{~mm}$, and iron content of $40 \mathrm{vol} \%$.

The measurement setup is shown in Fig. 3. An electromagnet consists of iron cores and a magnetic coil. A wire of diameter $1 \mathrm{~mm}$ is used to wind the coil in 800 turns. Two MREs are placed in the gaps between upper and lower cores of the electromagnet. In these gaps, a magnetic flux density was varied from $0 \mathrm{mT}$ to $326 \mathrm{mT}$ in response to a current change from 0 A to $6 \mathrm{~A}$, respectively. While the lower core is installed on a base exposed to excitation, the upper core is fixed along a load sensor. The base is excited by a shaker with excitation signal supplied by a signal generator and a power amplifier. The displacement of the base and upper core's force are measured using a laser displacement sensor and a load sensor, respectively. The force-displacement response is processed by a Fast Fourier Transform (FFT) spectrum analyzer. A direct current (DC) power supply provides adjustable direct current to a magnetic coil. In dynamic tests, numerous experiments were conducted for various harmonic inputs. The excitation frequency was adjusted from $1 \mathrm{~Hz}$ to $30 \mathrm{~Hz}$, excitation amplitude was changed from $0.4 \mathrm{~mm}$ to $1.4 \mathrm{~mm}$, 
and applied current was driven from 0 A to 6 A (magnetic flux density was adjusted from 0 mT to 326 $\mathrm{mT})$.

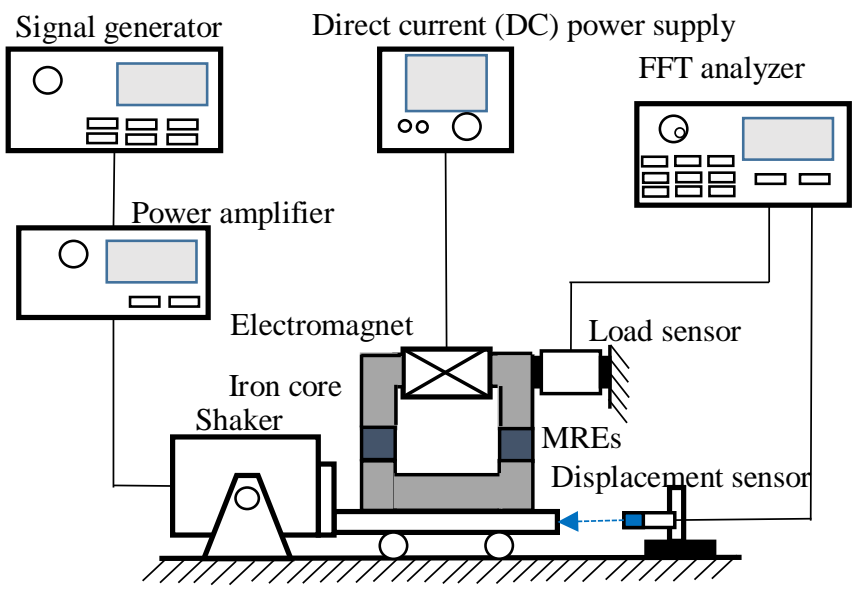

(a)

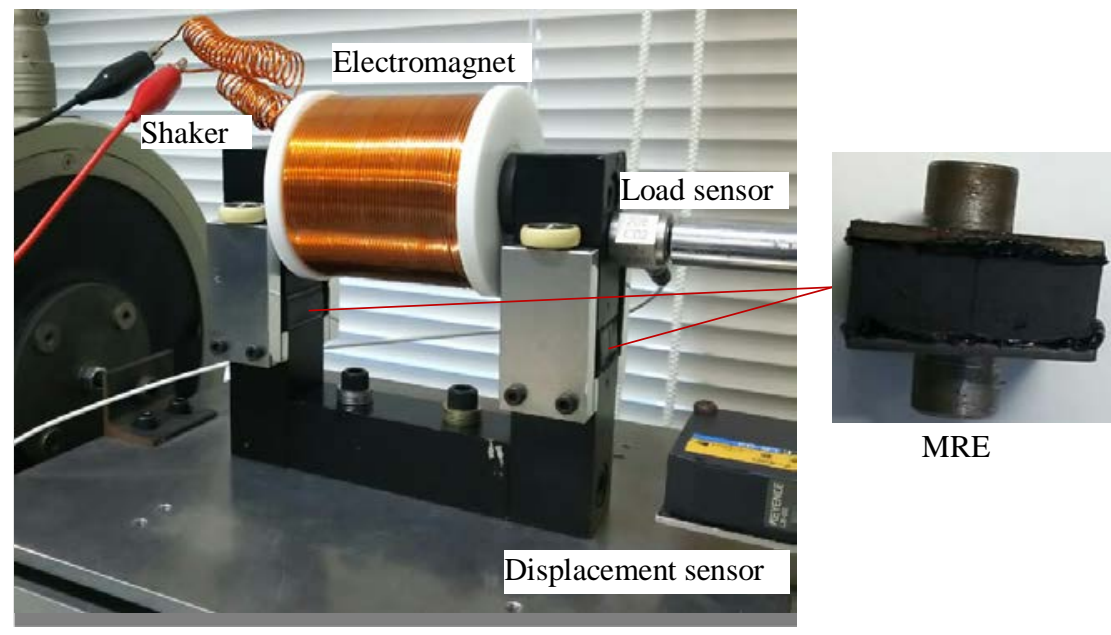

(b)

Fig. 3 MRE viscoelastic property measurement system: (a) schematic; (b) photograph.

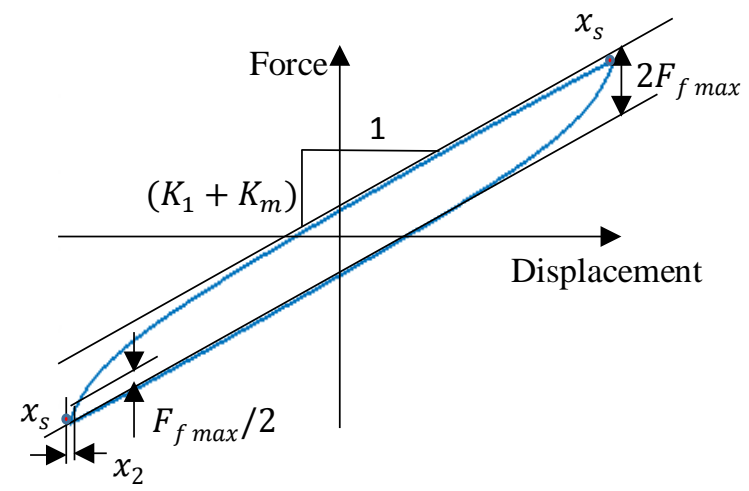

Fig. 4 Definitions of the model parameters: $K_{1}, K_{m}, F_{f \text { max }}$, and $x_{2}$. 


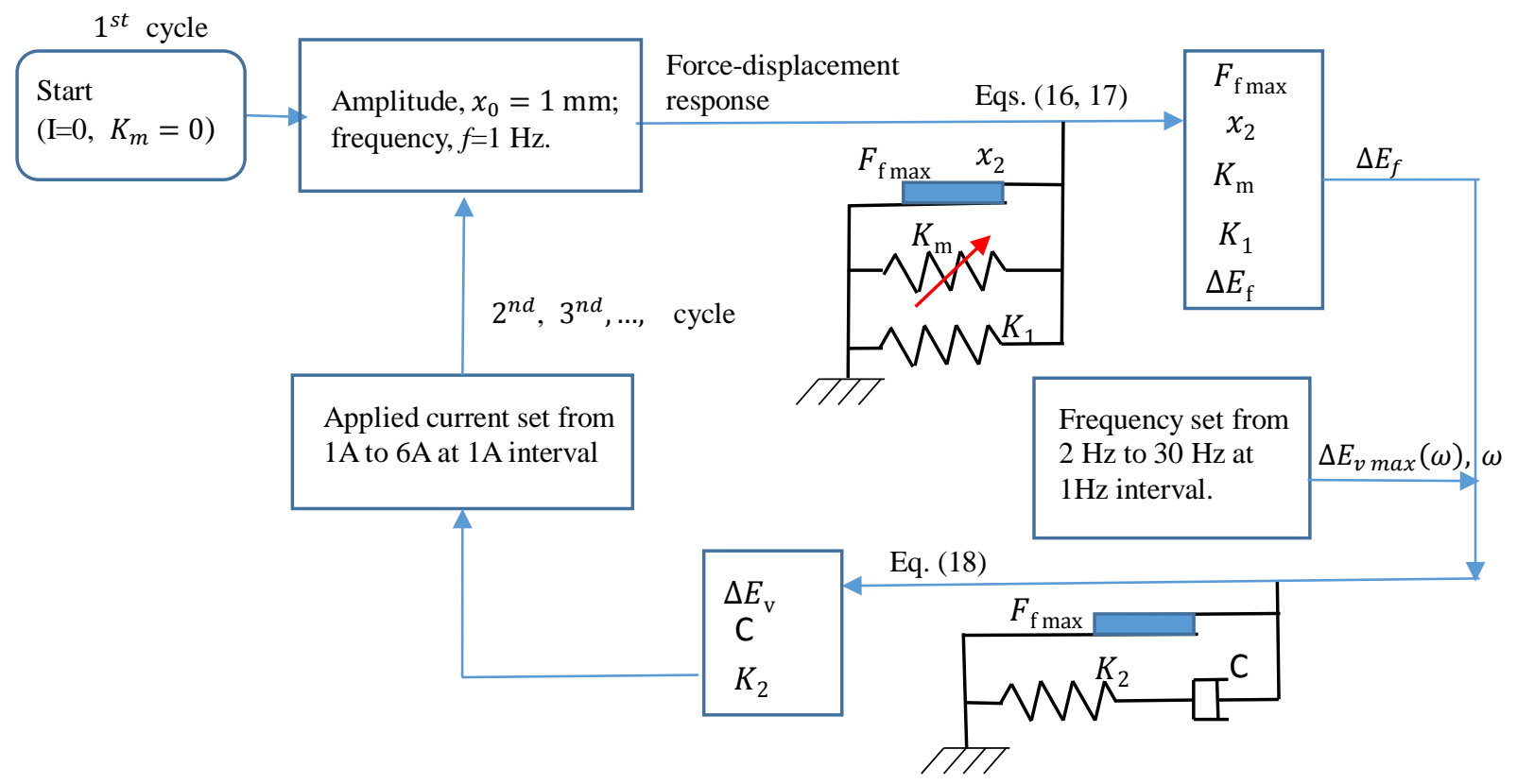

Fig. 5 Proposed identification steps of the model parameters in MRE modeling

Table 1 Parameters defined for different applied current values

\begin{tabular}{lllll}
\hline & $0 \mathrm{~A}$ & $2 \mathrm{~A}$ & $4 \mathrm{~A}$ & $6 \mathrm{~A}$ \\
\hline$K_{1}[\mathrm{~N} / \mathrm{mm}]$ & 13 & 13 & 13 & 13 \\
$K_{2}[\mathrm{~N} / \mathrm{mm}]$ & 9 & 9 & 9 & 9 \\
$\mathrm{C}[\mathrm{N} . \mathrm{s} / \mathrm{mm}]$ & 0.035 & 0.035 & 0.035 & 0.035 \\
$K_{m}[\mathrm{~N} / \mathrm{mm}]$ & 0 & 6.5 & 11 & 11.5 \\
$F_{\text {fmax }}[\mathrm{N}]$ & 2.4 & 6.5 & 9.5 & 10 \\
$x_{2}[\mathrm{~mm}]$ & 0.09 & 0.09 & 0.09 & 0.09 \\
\hline
\end{tabular}

\subsection{Determination of model parameters}

The parameters are determined according to the following procedure, from step 1 to step 3.

Step 1: Determination of the model parameters, $K_{1}, K_{m}, F_{f \text { max }}$, and $x_{2}$.

The displacement amplitude, $x_{0}=1 \mathrm{~mm}$ and excitation frequency, $f=1 \mathrm{~Hz}$, were selected for experiment. The force-displacement curve is shown in Fig. 4. In the case of low excitation frequency, the viscous effects modeled by Eqs. (2) and (5) become extremely small and can be neglected $\left(F_{2} \approx 0, E_{v} \approx 0\right)$. When displacement is $x \gg x_{s}$ or $x \ll x_{s}$ ( $x_{s}$ is the static equilibrium), the friction force, represented by Eq. (9), becomes maximum $\left(F_{f}=F_{f \max }\right)$. The total force and loss energy per cycle in Eqs. (11) and (12) are rewritten as,

$$
\begin{gathered}
F=\left(K_{1}+K_{m}\right) x+F_{f \max }, \\
\Delta E=\Delta E_{f},
\end{gathered}
$$


where $F(x)$ is the force determined by measured force-displacement loop, and $\Delta E$ is the loss energy per cycle determined by the area enclosed by the loop, $K_{1}$ is the nominal stiffness of MRE without magnetic field, and $K_{m}$ is the increment stiffness when the electric current is applied. Consequently, $K_{1}$, $K_{m}, F_{f \max }$, and $\Delta E_{f}$ are calculated, where $x_{2}$ is used to determine the rate of friction force development relative to the displacement. The parameters are illustrated in Fig. 4.

Step 2: Determination of the viscosity parameters, $K_{2}$ and $C$.

Under the constant displacement amplitude $x_{0}=1 \mathrm{~mm}$, frequency was varied from $2 \mathrm{~Hz}$ to $30 \mathrm{~Hz}$ at 1 $\mathrm{Hz}$ interval. From the experiments, the maximum loss energy $\Delta E_{\max }(\omega)$ is used for determining model parameters. From Eq. (10), the friction loss energy $\left(\Delta E_{f}\right)$ is found to be independent of excitation frequency. The viscous loss energy, modeled by Eq. (5), is dependent on frequency and reaches maximum at characteristic frequency $\omega=K_{2} / C$,

$$
\Delta E_{v \max }(\omega)=0.5 \pi \omega K_{2} x_{0}^{2}=\Delta E_{\max }(\omega)-\Delta E_{f},
$$

where $\Delta E_{\max }(\omega)$ and $\omega=K_{2} / C$ are the maximum loss energy and the characteristic frequency determined by experimental results. $\Delta E_{f}$ is determined by Eq. (17) in step 1 . The model viscosity parameters, $K_{2}$ and $C$ are then identified.

Step 3: Redo step 1 and step 2 for different applied currents.

The proposed procedures are schematically displayed in Fig. 5. The identified parameters are shown in Table 1. From the table, the model parameters such as $K_{1}, K_{2}, C$, and $x_{2}$ are found to change insignificantly by the applied currents. On the other hand, the parameters $K_{m}$ and $F_{f \max }$ increase significantly on increasing current. From the values in Table $1, K_{m}$ and $F_{f \max }$ are approximated by the following continuous function.

$$
K_{m}=-0.38 I^{2}+4.25 I, F_{f \max }=-0.24 I^{2}+2.75 I+2.4 \quad I \in[0,6]
$$

Consequently, the model parameter values and the approximation formulae were identified as listed in Table 2 .

Table 2 Parameter for the proposed MRE model

\begin{tabular}{ll}
\hline Stiffness $\left(K_{1}\right)$ & $13 \mathrm{Nmm}^{-1}$ \\
Stiffness $\left(K_{2}\right)$ & $10 \mathrm{Nmm}^{-1}$ \\
Viscous damping $(C)$ & $0.035 \mathrm{Nsm}^{-1}$ \\
Friction displacement $\left(x_{2}\right)$ & $0.09 \mathrm{~mm}$ \\
Maximum friction force $\left(\mathrm{F}_{\mathrm{f} \text { max }}\right)$ & $F_{f \max }=-0.24 I^{2}+2.75 I+2.4$ \\
Variable stiffness $\left(K_{\mathrm{m}}\right)$ & $K_{m}=-0.38 I^{2}+4.25 I$ \\
Applied current $(I)$ & $I \in[0,6]$ Ampere \\
\hline
\end{tabular}




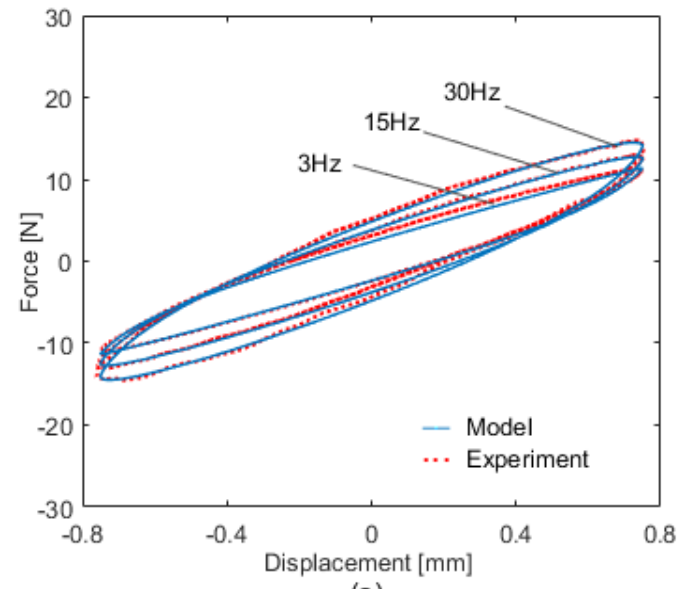

(a)

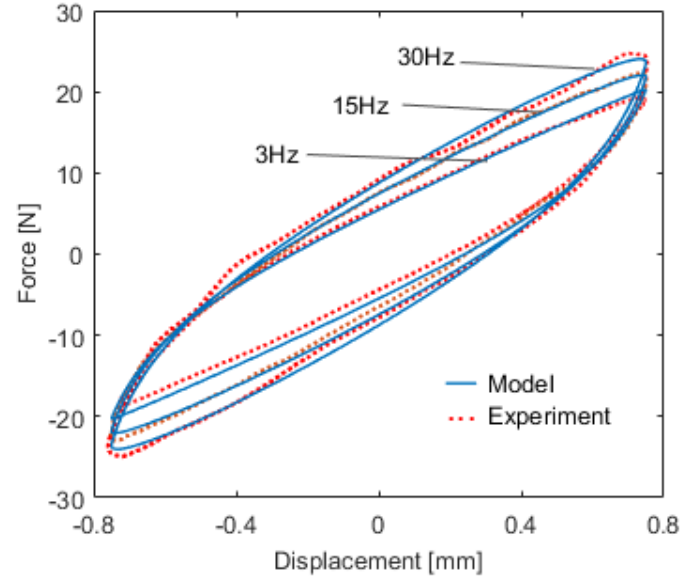

(b)

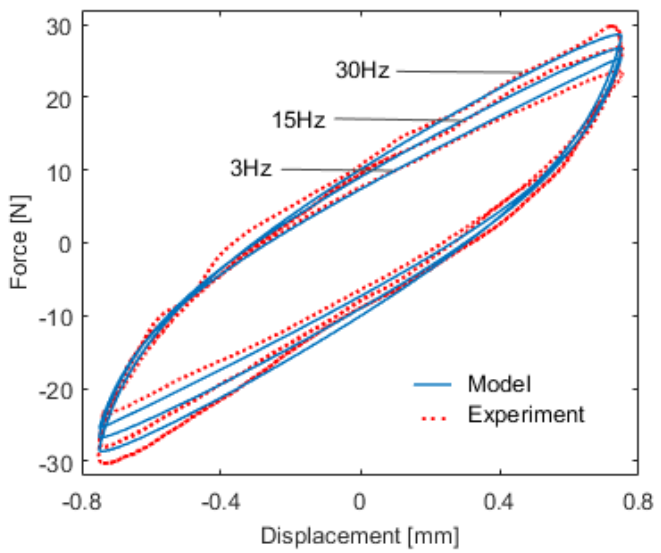

(c)

Fig. 6 Force-displacement response under different frequencies with excitation amplitude $x_{0}=0.75 \mathrm{~mm}$ : (a) $I=0 \mathrm{~A}(0 \mathrm{mT})$, (b) $I=2 \mathrm{~A}(218 \mathrm{mT})$, and (c) $I=4 \mathrm{~A}(267 \mathrm{mT})$.

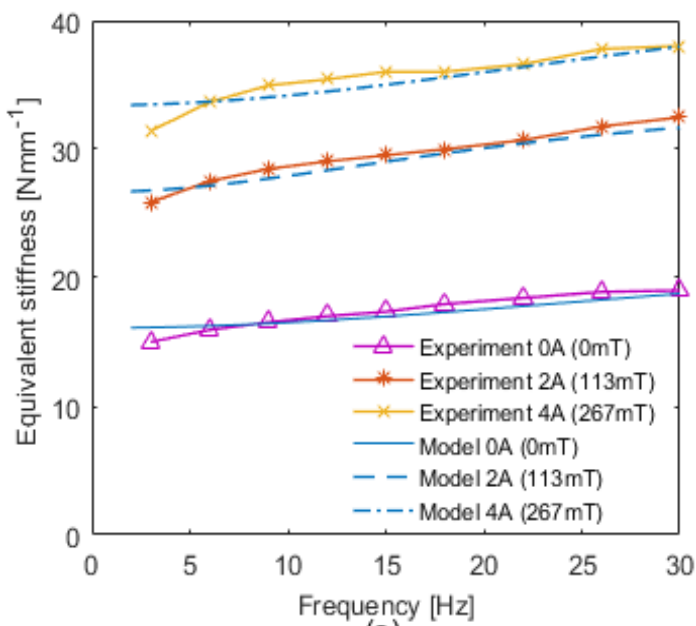

(a)

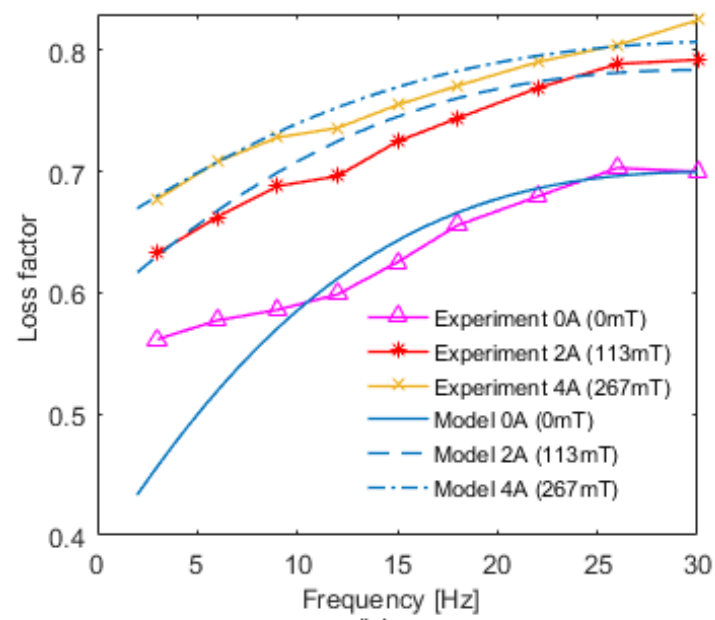

(b)

Fig. 7 Stiffness and loss factor versus excitation frequency for different applied currents with excitation amplititude $x_{0}=0.75 \mathrm{~mm}$ : (a) the equivalent stiffness and (b) the loss factor. 


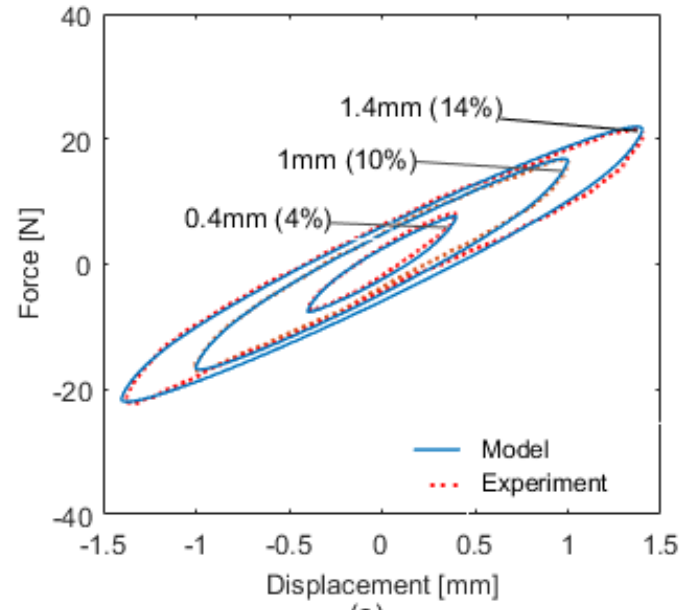

(a)

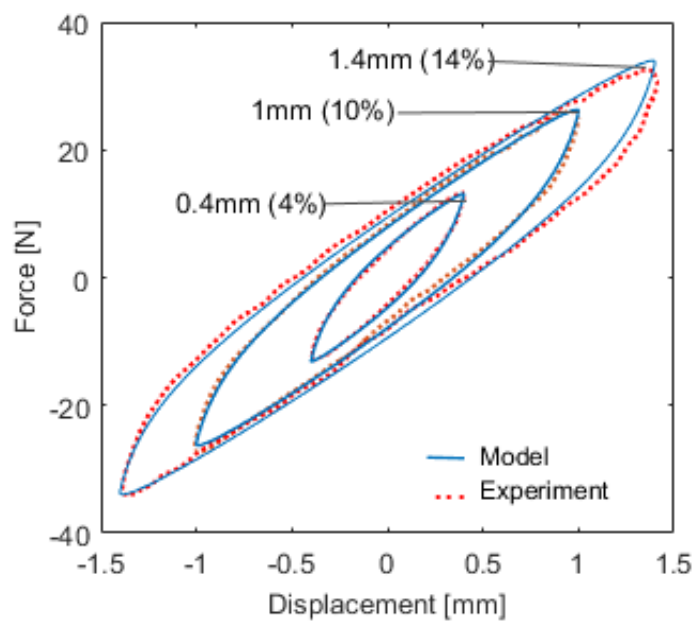

(b)

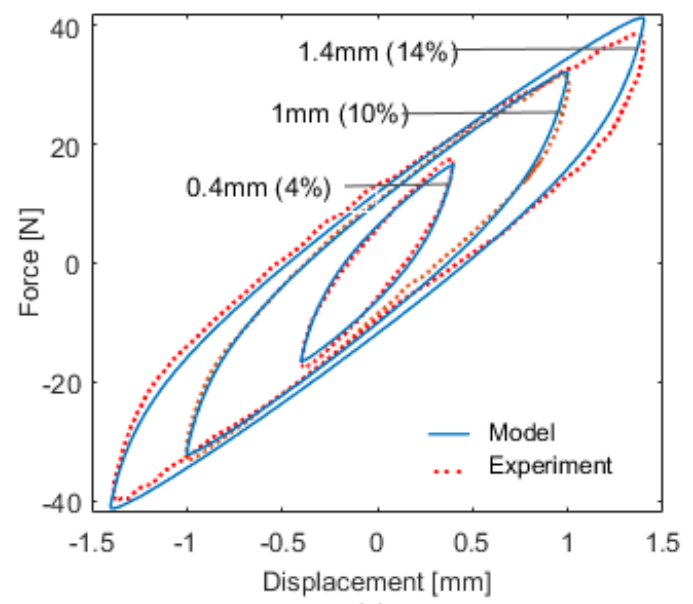

(c)

Fig. 8 Force-displacement response under different amplitude levels with excitation frequency $f=15 \mathrm{~Hz}$ :

(a) $I=0 \mathrm{~A}(0 \mathrm{mT})$, (b) $I=2 \mathrm{~A}(218 \mathrm{mT})$, and (c) $I=4 \mathrm{~A}(267 \mathrm{mT})$.

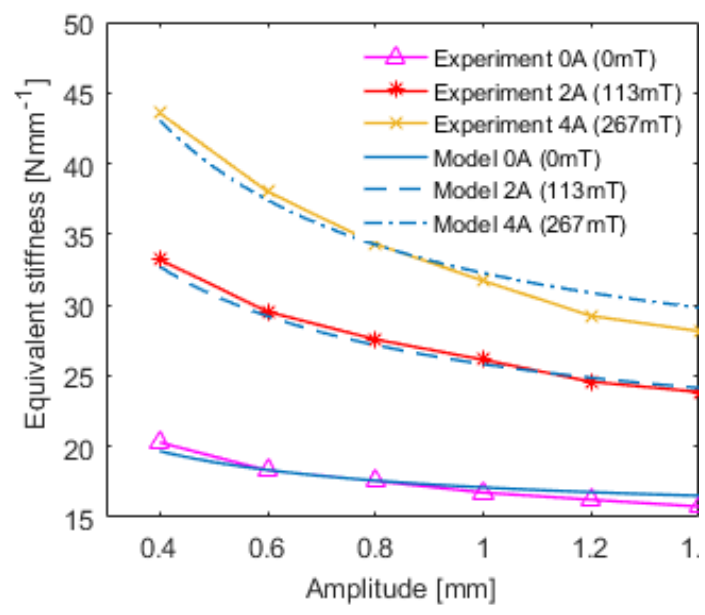

(a)

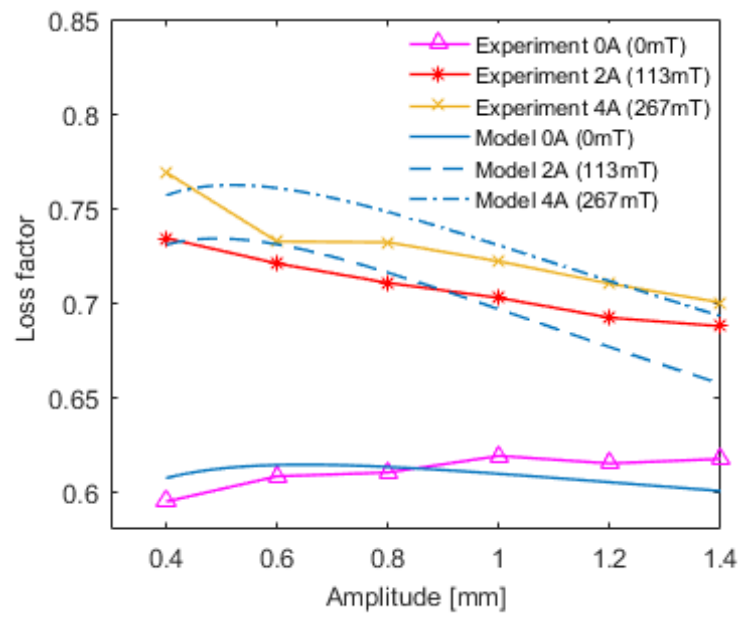

(b)

Fig. 9 Stiffness and loss factor versus excitation amplitude for different levels of applied current with excitation frequency $f=15 \mathrm{~Hz}$ : (a) the equivalent stiffness and (b) the loss factor. 


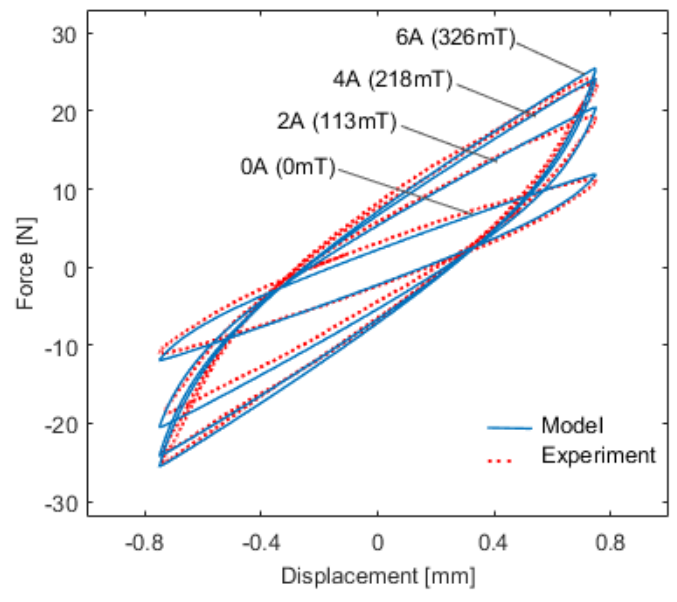

(a)

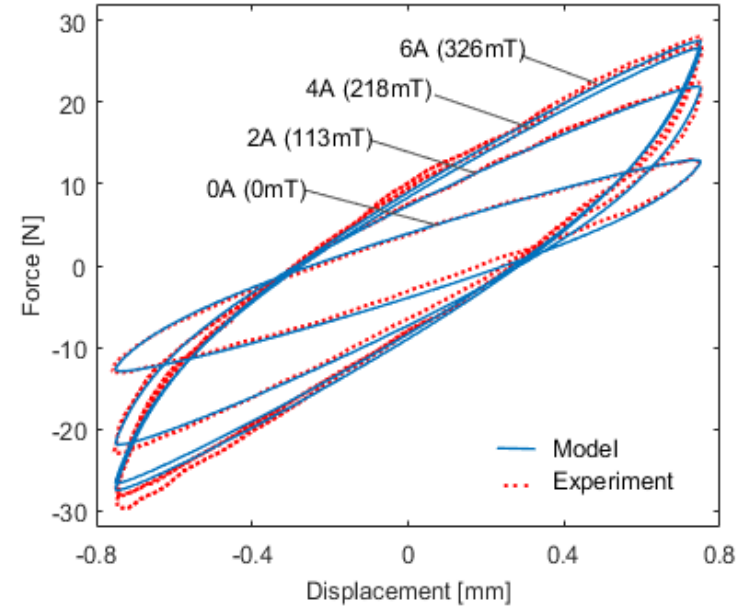

(b)

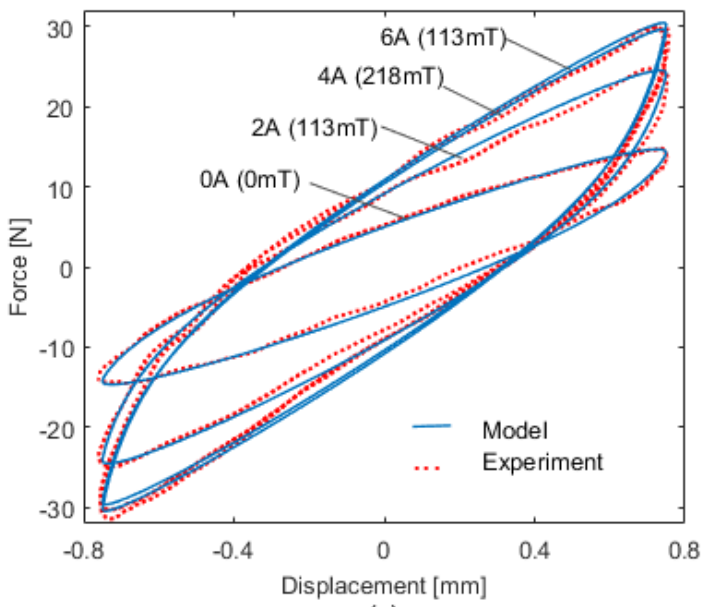

(c)

Fig. 10 Force-displacement response under different levels of applied current with excitation amplitude $x_{0}=0.75 \mathrm{~mm}$ : (a) $f=3 \mathrm{~Hz}$, (b) $f=15 \mathrm{~Hz}$, and (c) $f=30 \mathrm{~Hz}$.

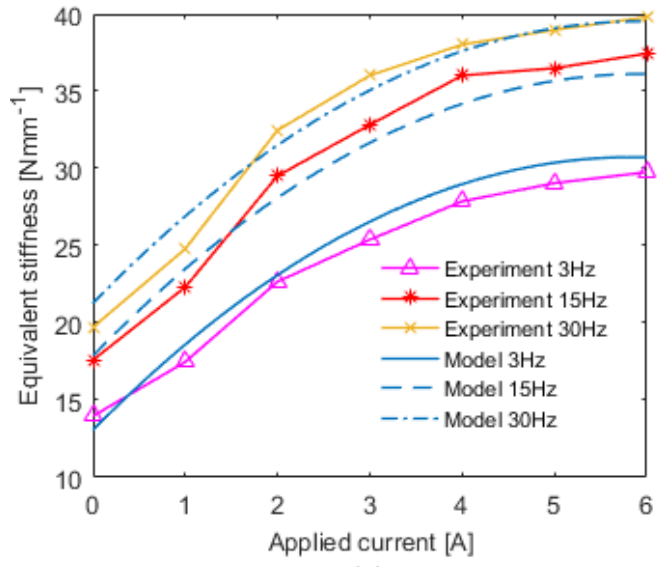

(a)

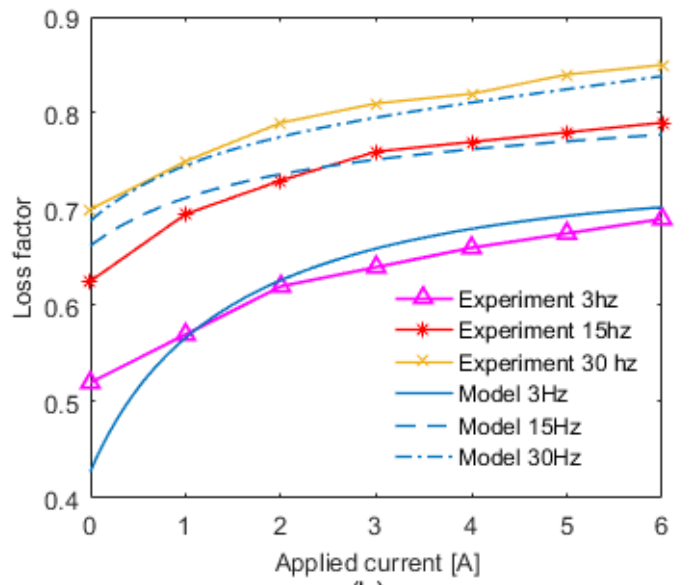

(b)

Fig. 11 Stiffness and loss factor versus applied current for different frequencies with excitation amplitude $x_{0}=0.75 \mathrm{~mm}$ : (a) the stiffness and (b) the loss factor. 
Table 3 Fitness of the proposed model in frequency dependency (values are presented in percentage).

\begin{tabular}{ccccc}
\hline $\begin{array}{c}\text { Frequency } \\
(\mathrm{Hz})\end{array}$ & $0 \mathrm{~A}$ & $2 \mathrm{~A}$ & $4 \mathrm{~A}$ & $6 \mathrm{~A}$ \\
\hline $1 \mathrm{~Hz}$ & 83.5 & 85.5 & 89.2 & 87.1 \\
$3 \mathrm{~Hz}$ & 85.6 & 89.0 & 92.4 & 90.2 \\
$6 \mathrm{~Hz}$ & 94.2 & 90.2 & 89.4 & 92.3 \\
$9 \mathrm{~Hz}$ & 96.3 & 98.2 & 96.9 & 97.6 \\
$12 \mathrm{~Hz}$ & 91.6 & 96.1 & 98.5 & 96.2 \\
$15 \mathrm{~Hz}$ & 90.2 & 94.2 & 96.1 & 97.3 \\
$18 \mathrm{~Hz}$ & 94.1 & 98.1 & 94.7 & 96.9 \\
$22 \mathrm{~Hz}$ & 92.6 & 97.0 & 91.0 & 97.3 \\
$26 \mathrm{~Hz}$ & 95.7 & 96.1 & 89.1 & 94.3 \\
$30 \mathrm{~Hz}$ & 94.3 & 94.2 & 90.4 & 92.2 \\
\hline
\end{tabular}

Table 4 Fitness of the proposed model in amplitude dependency (values are presented in percentage).

\begin{tabular}{lcccc}
\hline $\begin{array}{c}\text { Amplitude } \\
\text { (shear strain) }\end{array}$ & O A & 2 A & 4 A & $6 \mathrm{~A}$ \\
\hline $0.4 \mathrm{~mm} \mathrm{(4 \% )}$ & 85.1 & 91.0 & 91.1 & 89.3 \\
$0.6 \mathrm{~mm}(6 \%)$ & 89.2 & 92.8 & 92.7 & 91.4 \\
$0.8 \mathrm{~mm}(8 \%)$ & 92.9 & 93.4 & 94.0 & 94.2 \\
$1.0 \mathrm{~mm}(10 \%)$ & 94.9 & 95.7 & 95.9 & 94.1 \\
$1.2 \mathrm{~mm}(12 \%)$ & 95.4 & 96.5 & 95.6 & 95.6 \\
$1.4 \mathrm{~mm}(14 \%)$ & 91.8 & 94.7 & 93.6 & 92.5 \\
\hline
\end{tabular}

Table 5 Fitness of the proposed model in magnetic flux density dependency (values are presented in percentage).

\begin{tabular}{llccc}
\hline $\begin{array}{c}\text { Applied current } \\
\text { (magnetic flux density) }\end{array}$ & $1 \mathrm{~Hz}$ & $9 \mathrm{~Hz}$ & $18 \mathrm{~Hz}$ & $30 \mathrm{~Hz}$ \\
\hline 0 A (0 mT) & 83.5 & 97.3 & 94 & 94.1 \\
1 A (59 mT) & 86.1 & 96.6 & 94.3 & 93.4 \\
2 A (113 mT) & 85.2 & 98.2 & 98.1 & 94.2 \\
3 A (167 mT) & 87.4 & 96.4 & 95.2 & 92.7 \\
4 A (218 mT) & 89.2 & 96.9 & 94.7 & 90.4 \\
5 A (267 mT) & 90.5 & 96.7 & 96.1 & 93.1 \\
6 A (316 mT) & 87.1 & 97.3 & 96.9 & 92.7 \\
\hline
\end{tabular}

\subsection{Comparison of simulation and experimental results}

The proposed MRE model and relevant simulation results are compared with experimental results obtained by harmonic excitation. Three levels of displacement, three levels of input frequency, and four levels of magnetic field were arranged.

\subsubsection{Frequency dependency}

A displacement amplitude, $x_{0}=0.75 \mathrm{~mm}$, was set at excitation frequencies: $f=3,15$, and $30 \mathrm{~Hz}$. Measurements were performed in three levels of magnetic field: $0 \mathrm{mT}(0 \mathrm{~A}), 113 \mathrm{mT}$ (2 A), and $218 \mathrm{mT}$ (4 
A). The force-displacement loops are shown in Fig. 6. Overall agreement between measured and simulated loops is found. The loops tend to become elliptic as the frequency increases. The gradient of the main axis and the area of hysteresis loops become large as the external magnetic field increases. The smooth Coulomb friction model is adaptable in representing the rate-dependence of the force-displacement relationship. As shown in Fig. 7, the equivalent stiffness and the loss factor defined by Eqs. (14) and (15) fit well with those from the measurements. Both the equivalent stiffness and the loss factor increase gradually by the increment of the excitation frequency. In addition, both values have similar trends for different levels of current.

\subsubsection{Amplitude dependency}

Under a harmonic excitation with frequency $f=15 \mathrm{~Hz}$, three displacement amplitudes were provided: $x_{0}=0.4,0.8$, and $1.4 \mathrm{~mm}$. Measurements and simulations were performed for three levels of magnetic field strength: $0 \mathrm{mT}(0 \mathrm{~A}), 113 \mathrm{mT}$ (2 A), and $218 \mathrm{mT}$ (4 A). The force-displacement loops are compared in Fig. 8. The force-displacement loops obtained by the numerical model agree well with the experimental result. The slopes of hysteresis loops decrease with the increase in amplitude and this trend is similar for all values of magnetic fields. As shown in Fig. 9(a), the equivalent stiffness is well estimated by the proposed model. The stiffness decreases as excitation amplitude increases. The decreasing trend in the loss factor obtained by the proposed model is found to coincide with the measured one at an acceptable level, as shown in Fig. 9(b). The loss factor changes monotonically to the change in excitation amplitude. The similar trends are observed for all values of applied currents.

\subsubsection{Magnetic field dependency}

Under the displacement amplitude of $x_{0}=0.75 \mathrm{~mm}$, four levels of magnetic field were applied to the isolator: $0 \mathrm{mT}(\mathrm{A}), 113 \mathrm{mT}$ (2 A), $218 \mathrm{mT}$ (4 A), and $326 \mathrm{mT}$ (6 A). The measurements and simulations were performed for three different excitation frequencies: $3 \mathrm{~Hz}, 15 \mathrm{~Hz}$, and $30 \mathrm{~Hz}$. The force-displacement loops are shown in Fig. 10. A good agreement between measured and simulated loops can be found. The loops tend to become elliptic as the magnetic flux density increases. The difference between measured and simulated loops exhibits the same degree of error in different levels of magnetic flux density. Fig. 11 shows the comparisons of equivalent stiffness and loss factor between proposed model and measured results. A good agreement between numerical responses and experimental results is achieved. The same tendency is observed with different excitation frequency; the higher the excitation frequency, the higher the equivalent stiffness and the loss factor became. Under specific excitation frequency, the equivalent stiffness shows parabolic increase as the applied current is increased. The loss factor increases gradually with the increase in applied current. 


\subsubsection{Fitness value of the proposed model}

The fitness value is calculated by the following Eq. (20), using the normalized root mean square function [10].

$$
\text { Fitness value }(\%)=100 \times\left[1-\frac{\left\|F_{\text {Model }}-F_{\text {Exp }}\right\|}{\left\|F_{\text {Exp }}-\bar{F}_{\text {Exp }}\right\|}\right] \text {, }
$$

In Eq. (20), $\|\ldots\|$ is the norm function, $\ldots$ the mean function (average value), $F_{\text {Model }}$ the numerical force vector in one cycle, $F_{E x p}$ the experimental force vector in one cycle. These vectors have the same displacement vector in response.

The calculated fitness values in percentage are listed in Tables 3, 4, and 5 for the respective dependency tests. In most cases, the fitness is higher than $90 \%$. The average fitness is about $93 \%$. From Tables 3 and 4, it is evident that the proposed model works effectively within the frequency range of $3 \mathrm{~Hz}$ and $30 \mathrm{~Hz}$ and shear strain between $6 \%$ and 14\%. However, the model may provide inaccurate prediction results when working under low frequency and small amplitude excitation.

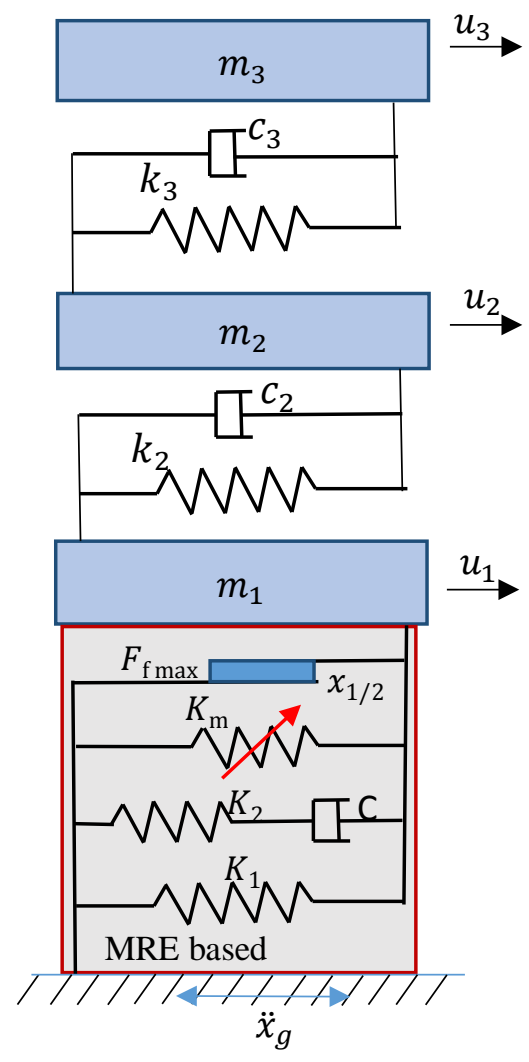


Fig. 12 Schematic of the two-story building with a fundament plate is rigidly connected by an MRE-base isolator.

Table 6 Scaling factor of the variables

\begin{tabular}{cccccc}
\hline Variables & Height & time & frequency & acceleration & mass \\
\hline Scaling factor & $1: 25$ & $1: 5$ & $5: 1$ & $1: 1$ & $1: 625$ \\
\hline
\end{tabular}

\section{SEMI-ACTIVE CONTROL STRATEGY}

\subsection{A model of the isolated building}

In order to evaluate the performance of MRE-based isolator, a two-story building structure $\left(m_{2}, m_{3}\right)$ with a fundament plate $\left(m_{1}\right)$ which is rigidly connected to the isolator is considered as shown in Fig. 12. The isolator is also connected rigidly to the ground.

The equation of motion can be written as follows,

$$
M \ddot{u}+C \dot{u}+K u=\Lambda f_{M R E}-M \Gamma \ddot{x}_{g} .
$$

In Eq. (21), $M, C$, and $K$ represent $(n \times n)$ mass, damping, and stiffness matrices, respectively; $u=$ $\left[\begin{array}{lll}u_{1} & u_{2} & u_{3}\end{array}\right]^{T}$ is the vector of the displacements of the masses relative to the ground; $f_{M R E}$ is the control force generated by an MRE-based isolator; $\ddot{x}_{g}$ is ground acceleration; $\Lambda=\left[\begin{array}{ccc}1 & 0 & 0\end{array}\right]^{T}$ is the matrix determined by the placement of control devices; $\Gamma$ is the column vector of ones. The mass of the floors are $m_{1}=3 \mathrm{~kg}, m_{2}=m_{3}=2 \mathrm{~kg}$. The stiffness and damping are determined from the real structure as $k_{2}=k_{3}=1.1 \times 10^{5} \mathrm{~N} \mathrm{~m}^{-1}$ and $c_{2}=c_{3}=2 \mathrm{~N} \mathrm{~s} \mathrm{~m}^{-1}$, respectively. The system matrices of a twostory shear building with a fundament plate are,

$$
M=\left[\begin{array}{ccc}
m_{1} & 0 & 0 \\
0 & m_{2} & 0 \\
0 & 0 & m_{3}
\end{array}\right], C=\left[\begin{array}{ccc}
c_{2} & -c_{2} & 0 \\
-c_{2} & c_{2}+c_{3} & -c_{3} \\
0 & -c_{3} & c_{3}
\end{array}\right], K=\left[\begin{array}{ccc}
k_{2} & -k_{2} & 0 \\
-k_{2} & k_{2}+k_{3} & -k_{3} \\
0 & -k_{3} & k_{3}
\end{array}\right] \text {. }
$$

Defining the state vectors as $z=\left[\begin{array}{ll}u & \dot{u}\end{array}\right]^{T}$, Eq. (21) can be written in state space form as follows,

$$
\dot{z}=A z+B f_{M R E}+E \ddot{x}_{g}
$$

where

$$
A=\left[\begin{array}{cc}
0 & I \\
-M^{-1} K & -M^{-1} C
\end{array}\right], B=\left[\begin{array}{c}
0 \\
M^{-1} \Lambda
\end{array}\right], E=\left[\begin{array}{c}
0 \\
-\Gamma
\end{array}\right] .
$$

The two-story building model is investigated with height scale factor of 1:25. The height of the model is $0.3 \mathrm{~m}$ that corresponds to a height of $7.5 \mathrm{~m}$ of the real two-story building. All variables and dimensions are scaled according to modeling laws [18, 3], and their values are summarized in Table 6. Four cases 
were investigated in both numerical evaluation and experiment: "fixed base" means that the building is fixed to the ground (2DOF); "passive off” means the isolated building that works with an isolator without applied current (0 A); "passive on" means the isolated building that works with an isolator applied by a current (5 A); "fuzzy control” means the isolated building that works with an isolator controlled by fuzzy algorithm.

\subsection{Semi-active fuzzy control}

The fuzzy logic controller is designed to determine the command applied current of the MRE-based isolator according to its relative velocity and relative displacement. The isolator regulates the viscoelastic force according to the applied current input. The block diagram of the controller is shown in Fig. 13.

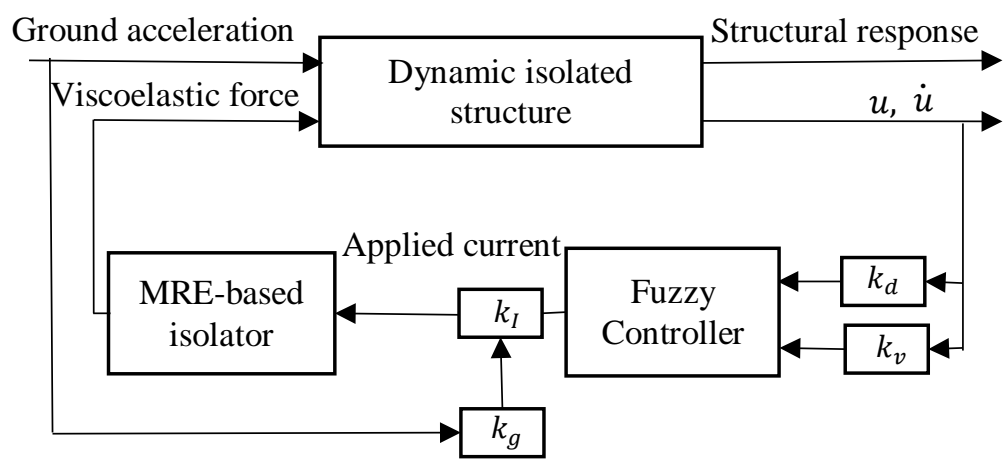

Fig. 13 Fuzzy control system

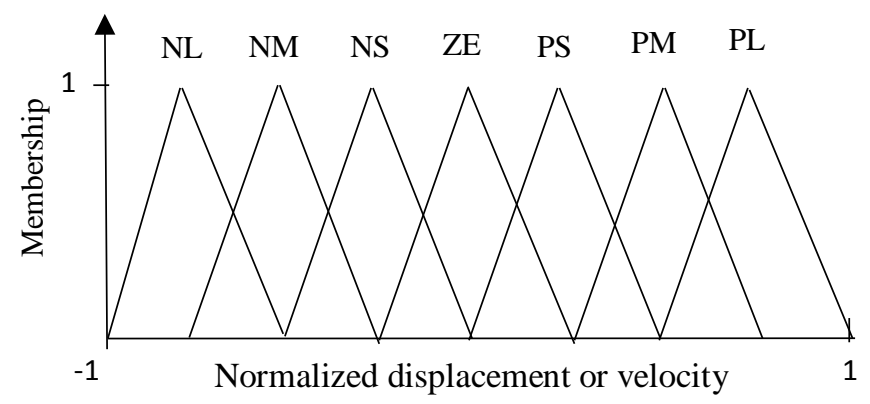

Fig. 14 Input membership functions

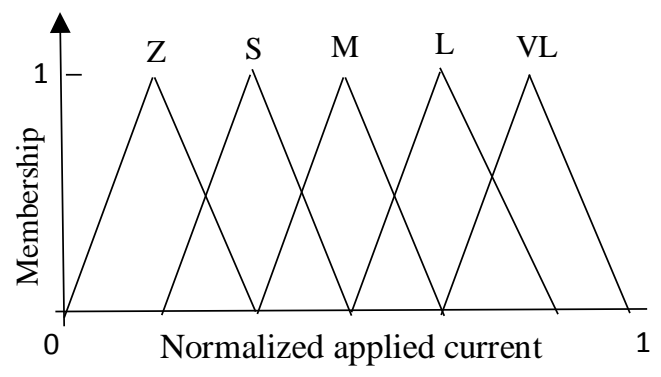

Fig. 15 Output membership functions 
The relative displacement and relative velocity of the fundament plate to the ground are selected as two input variables (Fig. 14), and command applied current is employed as a single output variable (Fig. 15). The definitions of the membership function of input variables are as follows: negative large (NL); negative medium (NM); negative small (NS); zero (ZE); positive small (PS); positive medium (PM); positive large (PL). For the electric current output, the control output functions are as follows: zero (ZE); small (S); medium (M); large (L); and very large (VL). The membership functions are structured in the shape of a triangle with Mamdani-type inference system; the center of gravity method is used for defuzzification. The rules are based on the skyhook on/off algorithm shown in Table 7.

The fuzzification factors used to convert the inputs into fuzzy variables are defined as $k_{d}$ and $k_{v}$, for the relative displacement and the relative velocity, respectively. The de-fuzzification factor used to convert the output is $k_{I}$. From the best results among considerable cases studied, $k_{I}$ seems to be strongly related to the ground acceleration.

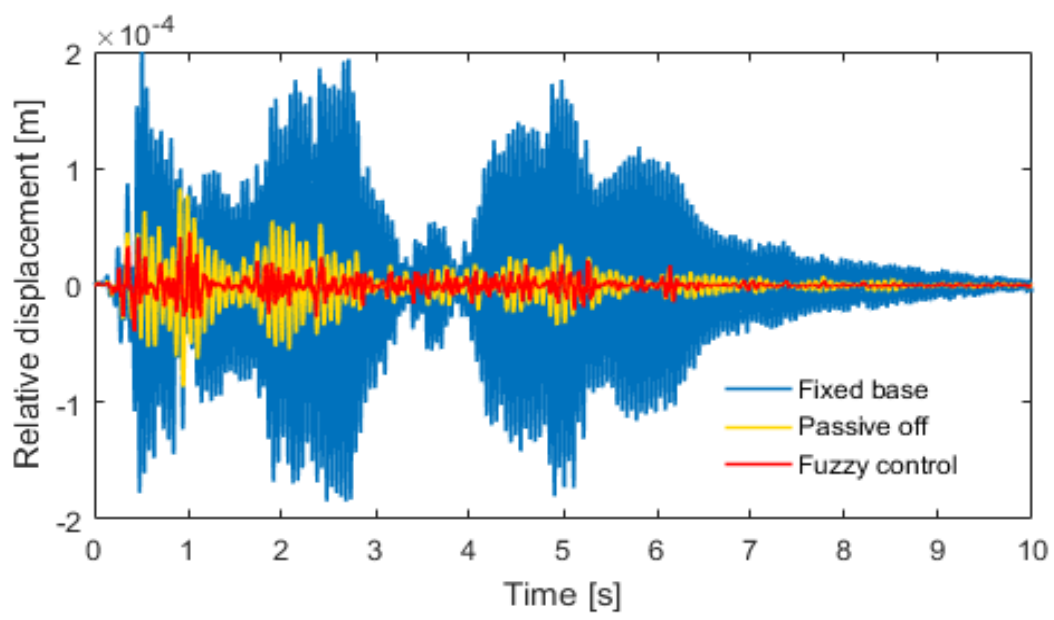

(a)

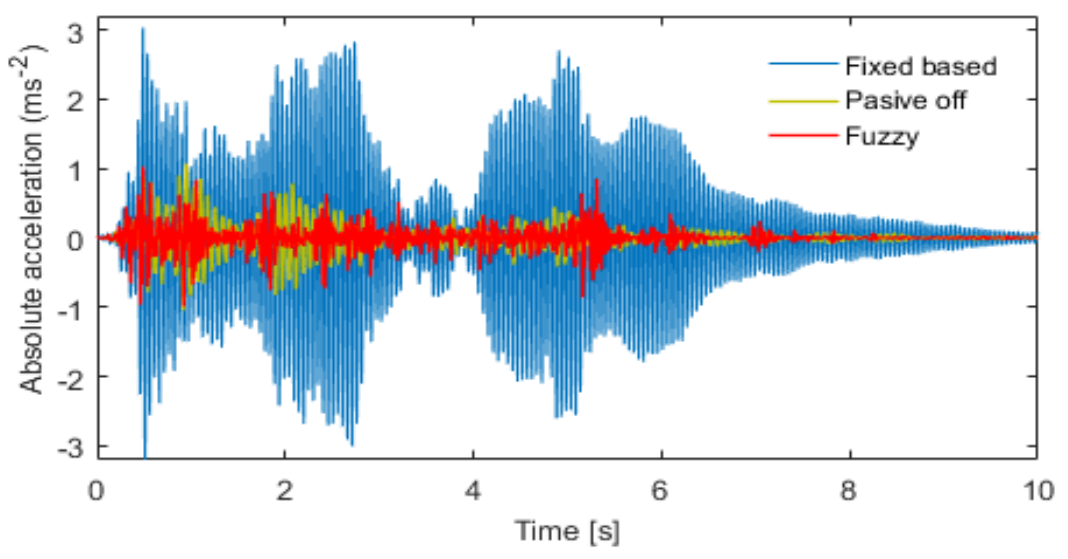

(b) 
Fig. 16 The relative displacement and absolute acceleration responses under El Centro earthquake: (a) the relative displacement response of the third mass to the fundament plate (the first mass), and (b) the absolute acceleration response in the third mass.

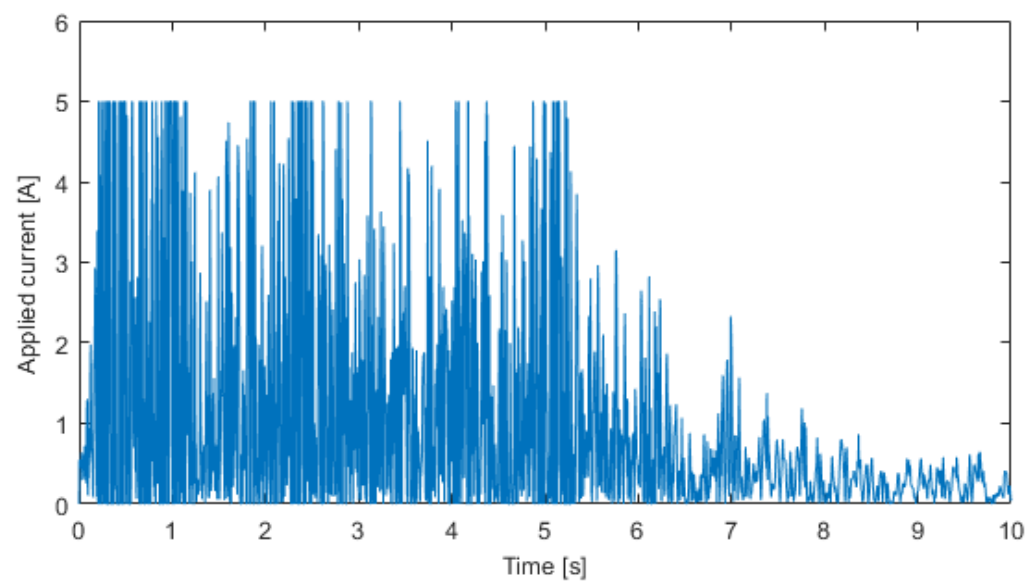

Fig. 17 Applied current for MRE-based isolator under El Centro earthquake

Table 7 Fuzzy inference rule

\begin{tabular}{cccccccc}
\hline & $u$ & & & & & & \\
$\dot{u}$ & $\mathrm{NL}$ & $\mathrm{NM}$ & $\mathrm{NS}$ & $\mathrm{ZE}$ & $\mathrm{PS}$ & $\mathrm{PM}$ & $\mathrm{PL}$ \\
\hline $\mathrm{NL}$ & $\mathrm{VL}$ & $\mathrm{VL}$ & $\mathrm{L}$ & $\mathrm{L}$ & $\mathrm{M}$ & $\mathrm{S}$ & $\mathrm{ZE}$ \\
$\mathrm{NM}$ & $\mathrm{VL}$ & $\mathrm{VL}$ & $\mathrm{L}$ & $\mathrm{M}$ & $\mathrm{S}$ & $\mathrm{ZE}$ & $\mathrm{S}$ \\
$\mathrm{NS}$ & $\mathrm{VL}$ & $\mathrm{L}$ & $\mathrm{M}$ & $\mathrm{S}$ & $\mathrm{ZE}$ & $\mathrm{S}$ & $\mathrm{M}$ \\
$\mathrm{ZE}$ & $\mathrm{L}$ & $\mathrm{M}$ & $\mathrm{S}$ & $\mathrm{ZE}$ & $\mathrm{S}$ & $\mathrm{M}$ & $\mathrm{L}$ \\
$\mathrm{PS}$ & $\mathrm{M}$ & $\mathrm{S}$ & $\mathrm{ZE}$ & $\mathrm{S}$ & $\mathrm{M}$ & $\mathrm{L}$ & $\mathrm{VL}$ \\
$\mathrm{PM}$ & $\mathrm{S}$ & $\mathrm{ZE}$ & $\mathrm{S}$ & $\mathrm{M}$ & $\mathrm{L}$ & $\mathrm{VL}$ & $\mathrm{VL}$ \\
$\mathrm{PL}$ & $\mathrm{ZE}$ & $\mathrm{S}$ & $\mathrm{M}$ & $\mathrm{L}$ & $\mathrm{L}$ & $\mathrm{VL}$ & $\mathrm{VL}$ \\
\hline
\end{tabular}

Table 8 Evaluated performance indices due to El Centro earthquake (RMS: root mean square; disp.: relative displacement of the third mass to fundament plate; acc.: absolute acceleration of the third mass).

\begin{tabular}{lllll}
\hline Control strategy & RMS disp. $(\mathrm{mm})$ & Max. disp. $(\mathrm{mm})$ & RMS acc. $\left(\mathrm{ms}^{-2}\right)$ & Max. acc. $\left(\mathrm{ms}^{-2}\right)$ \\
\hline Fixed based & $0.062(1)$ & $0.21(1)$ & $0.94(1)$ & $3.03(1)$ \\
Passive-off & $0.017(0.27)$ & $0.075(0.36)$ & $0.21(0.22)$ & $1.07(0.35)$ \\
Passive-on & $0.011(0.18)$ & $0.06(0.28)$ & $0.31(0.32)$ & $1.2(0.39)$ \\
Fuzzy & $0.009(0.15)$ & $0.05(0.24)$ & $0.18(0.17)$ & $1.02(0.33)$ \\
\hline
\end{tabular}

3.3 Numerical evaluation of the control performance

The performances of the "fixed base," "passive off," and "fuzzy control” are compared for the scaled El Centro earthquake. The responses of the scaled building to the scaled earthquake are shown in Figs. 16, 17 and Table 8. The relative displacement response of the third mass to the fundament plate is significantly reduced in the case of the isolated building in comparison to the "fixed base" (Fig. 16a). The "fuzzy 
control” performs remarkably better than the "passive off." In particular, the third mass absolute acceleration response is significantly suppressed in the system with MRE-based isolator (Fig. 16(b)). The "fuzzy control" performs moderately better than the "passive off" case in reducing the acceleration response. Fig. 17 shows the command applied current supplied to the MRE-based isolator with fuzzy controller. In the case of large displacement, the current supplied to the isolator switches between $0 \mathrm{~A}$ and 5 A. In the case of small displacement, the fuzzy controller produces a sufficiently necessary applied current for control. Time integral of the applied current signal indicates that the energy consumption in the "fuzzy control" is reduced by $65 \%$ in comparison to the energy required in the case of "passive on."

The RMS and maximum values of the third mass response are listed in Table 8 . The values in parentheses represent the ratio of the values to those obtained for the fixed building. The RMS ratio of the relative displacement response of third mass to the fundament plate in the case of "fuzzy control" is significantly reduced to 0.15 for the El Centro earthquake. The absolute acceleration RMS values of third mass are also decreased. In addition, the ratio of the maximum relative displacement and absolute acceleration response decrease to 0.24 and 0.33 ; all tested cases have minimum values.

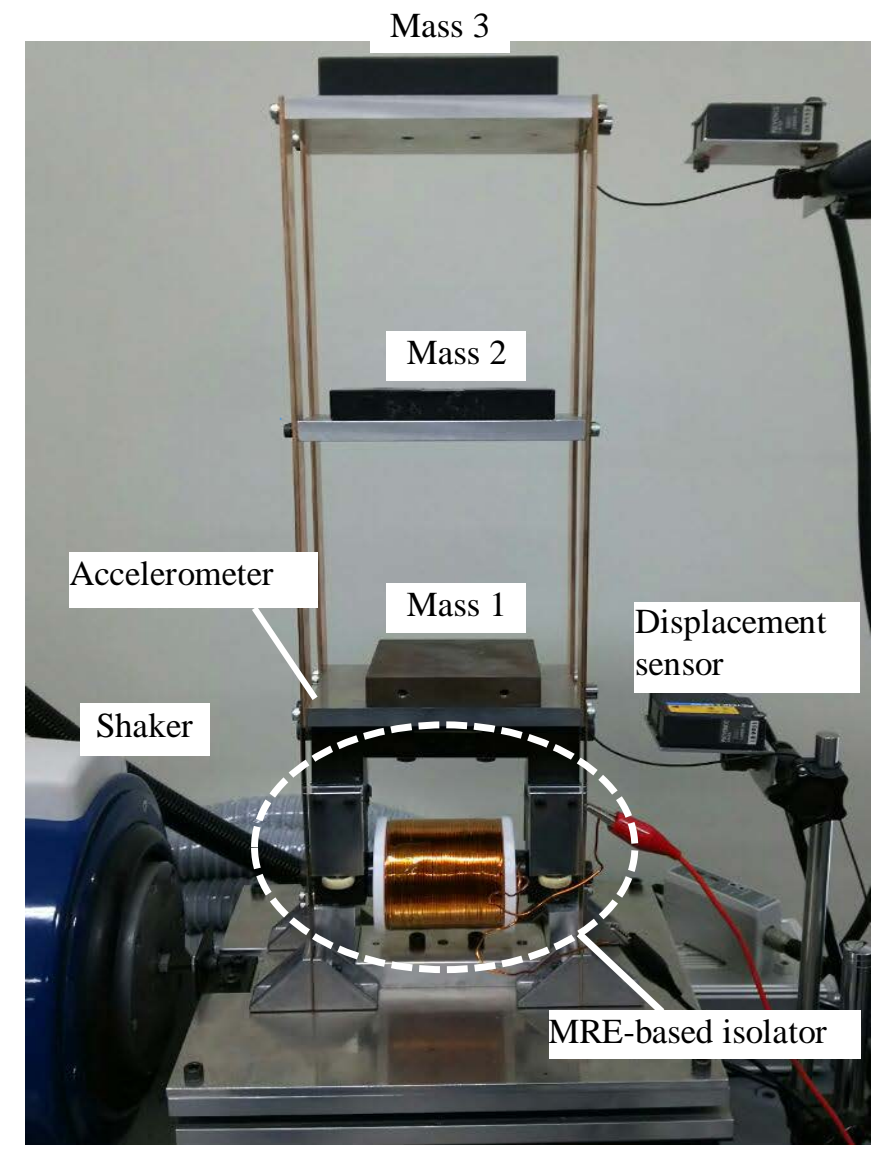

Fig. 18 Experimental setup: The building consist of two story (corresponding mass 2 and mass 3 ) and the MRE-based isolator is connected to the building model by the fundament plate (mass 1). 


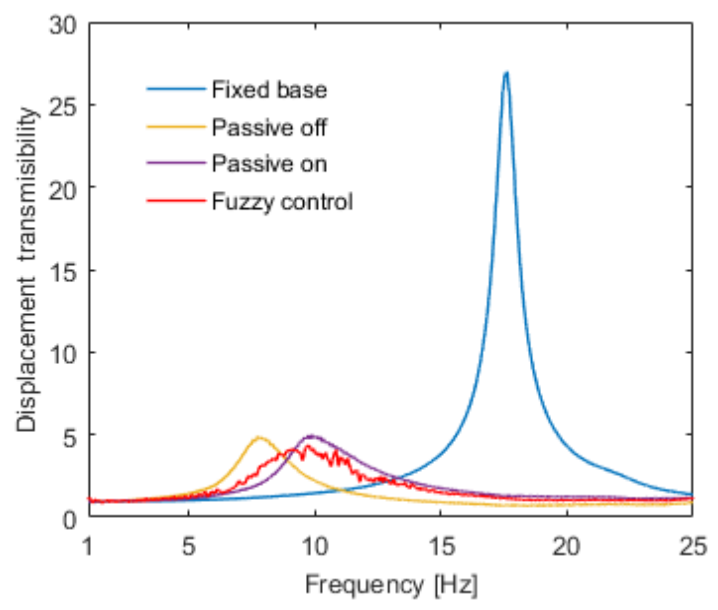

(a)

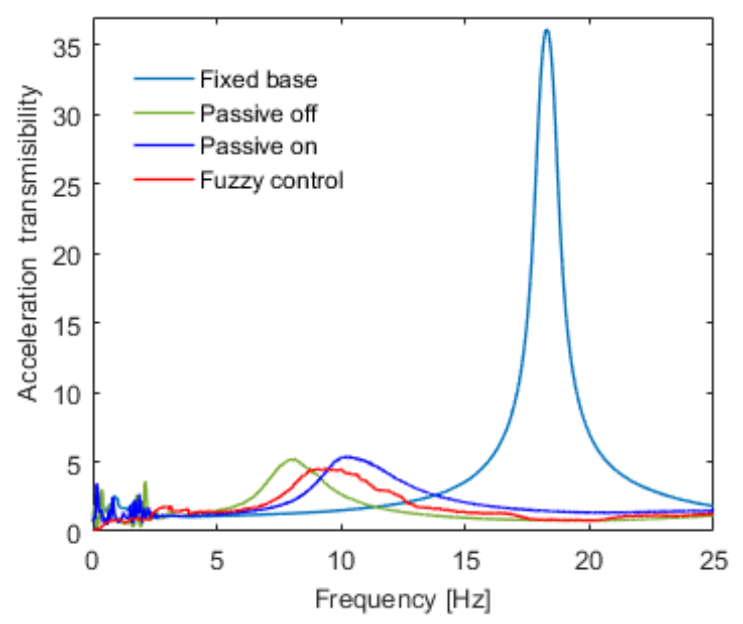

(b)

Fig. 19 Transmissibility of the scaled building: (a) displacement transmissibility and (b) acceleration transmissibility.

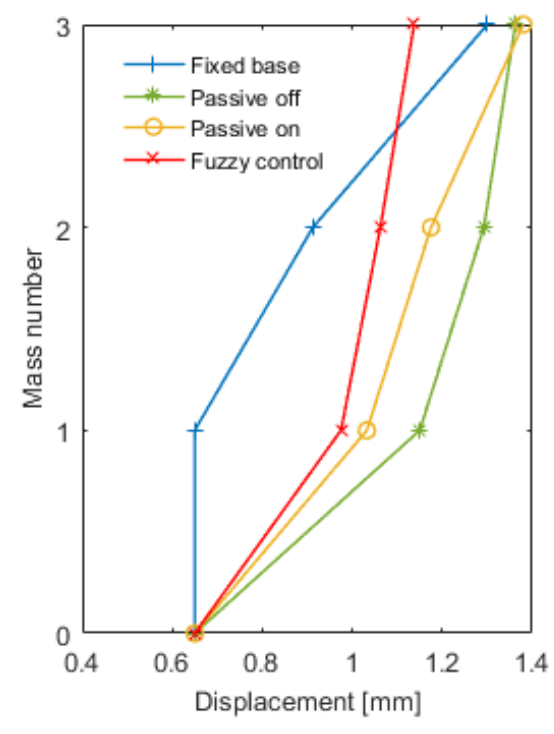

(a)

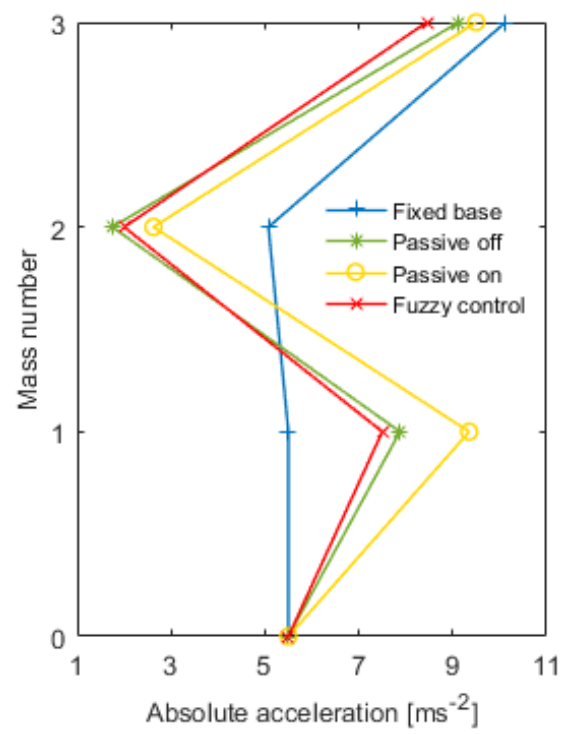

(b)

Fig. 20 Maximum displacement and absolute acceleration of scaled building: (a) displacement and (b) absolute acceleration.

\section{EXPERIMENTAL RESULTS}

Fig. 18 shows a photo of a scaled building (1:25). The displacement sensors and accelerometers are installed and calibrated on each floor of the scale building and shaking table. The analog displacement signals are sent to a digital signal processor (TMS 320 C6713 DSK Board). The fuzzy algorithm explained in section 3.2 is embedded in this processor to determine the command applied current. A high speed bi- 
polar power supply (Matsusada Precision Inc., Series POP 65-5) is used for the experiments such that the actual current precisely tracks the command current for the MRE-based isolator in real time. The sweptsine and random excitation were used to excite the table.

The experimental results are shown in Figs. 19-23 and Tables 9 and 10. Fig. 19 shows the displacement and acceleration transmissibility of the third mass responding to swept-sine excitation. The transmissibility is reduced significantly in the isolated building. The first natural frequency of the isolated building shifts from $7.6 \mathrm{~Hz}$ to $10.1 \mathrm{~Hz}$. Furthermore, the efficiency of isolation is improved using a fuzzy controller.

The maximum displacements and absolute accelerations are shown in Fig. 20. In "fuzzy control," the maximum displacement and the absolute acceleration of superstructure are decreased. Particularly, Fig. 21 shows the maximum values of the relative displacement. Better performances can be found in the isolated building than in the case of the fixed building. The isolator with fuzzy controller achieves the best performance. In Tables 9 and 10, numbers in parentheses denote the ratio of peak response and root mean square (RMS) values of the structure to those obtained for "fixed base" case. The peaks of the third mass displacement relative to the ground $\left(u_{3}\right)$ and the absolute acceleration $\left(\ddot{u}_{3}\right)$ are reduced by $15 \%$ and $24 \%$ respectively in "fuzzy control" compared to the "fixed base." The maximum relative displacement $\left(u_{3}-u_{1}\right)$ also decreases by $30 \%$ for "fuzzy control." Moreover, the root mean square (RMS) values also reduce holistically for the structure equipped with the isolator with fuzzy controller.

Fig. 22 shows time histories of the third mass absolute acceleration response; Fig. 23 shows the histories of the relative displacement response of the third mass to the fundament plate. It is evident that the responses are remarkably suppressed in the case of "fuzzy control." These responses significantly affect the destruction of the system under seismic events. Therefore, the application of MRE-based isolator with fuzzy controller is a promising measure to protect the building from collapse or fracture. 


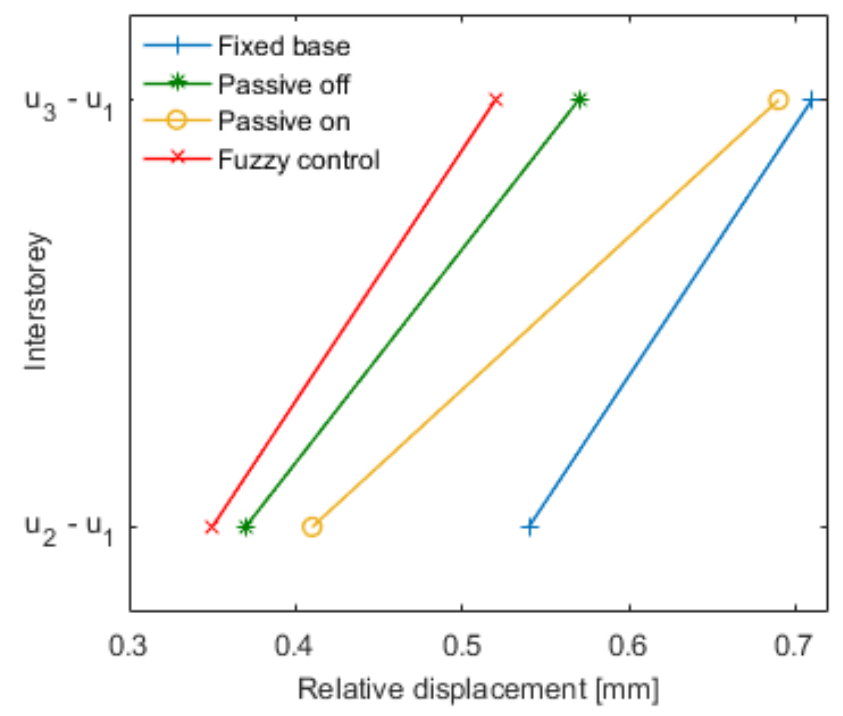

Fig. 21 Maximum values of relative displacement.

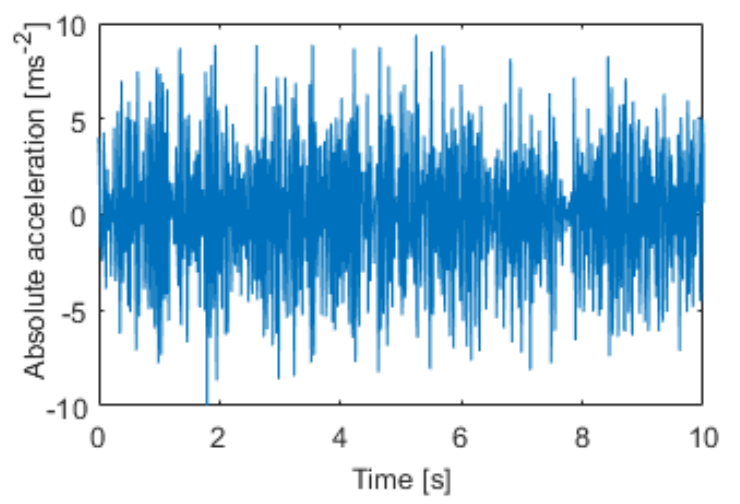

(a)

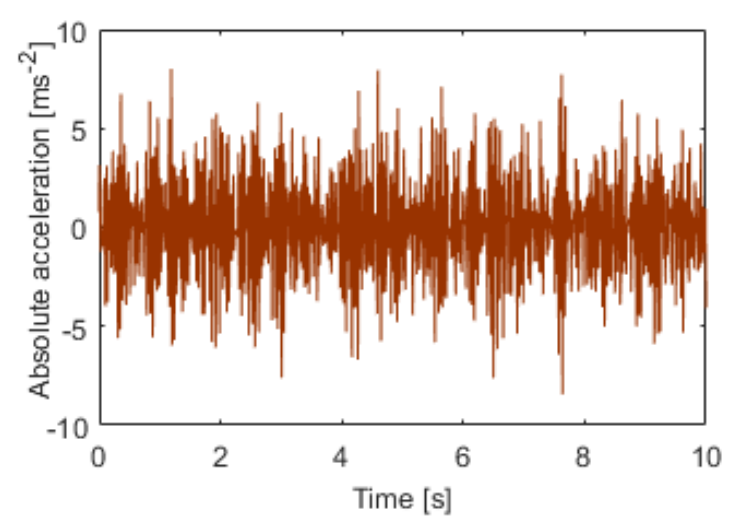

(b)

Fig. 22 The absolute acceleration of the third mass: (a) fixed base and (b) fuzzy control.

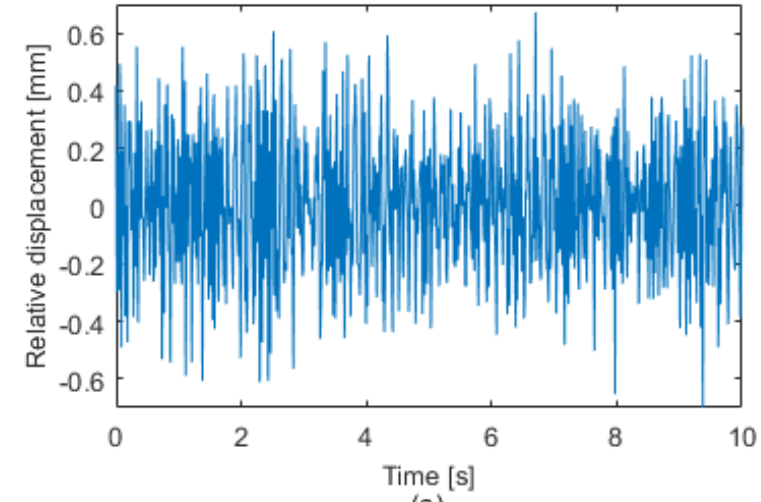

(a)

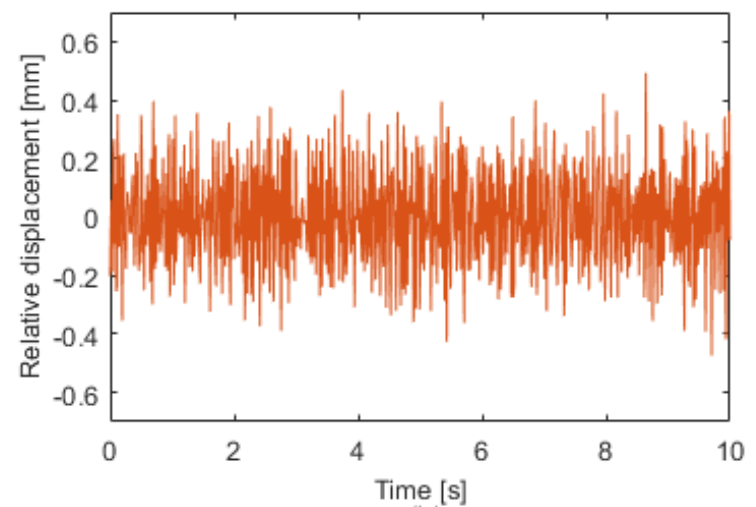

(b)

Fig. 23 The relative displacement of the third mass to the fundament plate (first mass): (a) fixed base and (b) fuzzy control. 
Table 9 Peak displacement of the third mass to the ground $\left(u_{3}\right)$, peak absolute acceleration of the third mass $\left(\ddot{u}_{3}\right)$, and peak relative displacement $\left(u_{3}-u_{1}\right)$ obtained in random excitation. Numbers in parentheses represent the ratio of peak value to that obtained in the case of "Fixed base."

\begin{tabular}{lllll}
\hline Control strategy & Fixed base & Passive off & Passive on & Fuzzy control \\
\hline$u_{3}(\mathrm{~mm})$ & $1.30(1)$ & $1.36(1.05)$ & $1.38(1.06)$ & $1.11(0.85)$ \\
$\ddot{u}_{3}\left(\mathrm{~ms}^{-2}\right)$ & $11.10(1)$ & $9.12(0.82)$ & $9.50(0.85)$ & $8.45(0.76)$ \\
$u_{3}-u_{1}(\mathrm{~mm})$ & $0.67(1)$ & $0.57(0.85)$ & $0.71(1.05)$ & $0.47(0.70)$ \\
\hline
\end{tabular}

Table 10 Root mean square (RMS) displacement of the third mass to the ground $\left(u_{3}\right)$, RMS absolute acceleration of the third mass $\left(\ddot{u}_{3}\right)$, and RMS relative displacement $\left(u_{3}-u_{1}\right)$ obtained in random excitation. Numbers in parentheses represent the ratio of RMS value to that obtained in the case of "Fixed base.”

\begin{tabular}{lllll}
\hline Control strategy & Fixed base & Passive off & Passive on & Fuzzy control \\
\hline$u_{3}(\mathrm{~mm})$ & $0.45(1)$ & $0.41(0.91)$ & $0.40(0.88)$ & $0.38(0.84)$ \\
$\ddot{u}_{3}\left(\mathrm{~ms}^{-2}\right)$ & $3.51(1)$ & $2.90(0.82)$ & $3.03(0.86)$ & $2.85(0.81)$ \\
$u_{3}-u_{1}(\mathrm{~mm})$ & $0.21(1)$ & $0.19(0.90)$ & $0.23(1.09)$ & $0.169(0.80)$ \\
\hline
\end{tabular}

\section{CONCLUSIONS}

In this study, the MRE-based isolator was developed for mitigating vibrations in a structure under seismic excitation. The dynamic viscoelastic model of the MRE-based isolator was presented, and a procedure to determine the six model parameters was introduced. The force-displacement relationship obtained by the numerical model is nearly consistent with the measurement results. Moreover, the proposed model predicts with high accuracy the dynamic viscoelastic characteristics of MRE in a wide range of frequencies $(3-30 \mathrm{~Hz}$ ) and shear strain (4\%-16\%). The MRE properties are strongly nonlinear functions of magnetic flux density, displacement amplitude, and the excitation frequency. Vibration responses of a scaled two story building were investigated. Using MRE-based isolator, the transmissibility of the building is reduced significantly and the first natural frequency shifts from $7.6 \mathrm{~Hz}$ to $10.1 \mathrm{~Hz}$ by switching applied current. A fuzzy control algorithm was used to enhance performance of the MRE-based isolator. Both numerical simulation and experimental results show that the "fuzzy control" provides better performance than "fixed base," "passive off," and "passive on.” The peaks of the third mass displacement 
and absolute acceleration are reduced by $15 \%$ and $24 \%$, respectively. The maximum relative displacement between third mass and fundament plate also decreases by 30\%. The MRE-based isolator used in conjunction with the fuzzy controller is efficient for mitigating vibrations in a two story building.

\section{ACKNOWLEDGMENTS}

The work was supported by the Japan Society for the Promotion of Science (JSPS) KAKENHI, Grantin-Aid for Scientific Research (B), Grant Number 15 H03936.

\section{REFERENCES}

[1] T. Komatsuzaki, Y. Iwata, Design of a real-time adaptively tuned dynamic vibration absorber with a variable stiffness property using magnetorheological elastomer, Shock and Vibration, vol. 2015, Article ID 676508, 11 pages 2015.

[2] G. J. Liao, X. L. Gong, S. H. Xuan, C. J. Kang, L. H. Zong, Development of a real-time tunable stiffness and damping vibration isolator based on magnetorheological elastomer, Journal of Intelligent Material Systems and Structures, Vol. 23, no. 1, pp. 25-33, 2012.

[3] J. Yang, S. Sun, T. Tian, W. Li, H. Du, G. Alici, M. Nakano, Development of a novel multi-layer MRE isolator for suppression of building vibrations under seismic, Mechanical Systems and Signal Processing, 70-71 (2016), pp. 811-820.

[4] H. J. Jung, S. H. Eem, D. D. Jang, J. H. Koo, Seismic performance analysis of a smart base-isolation system considering dynamics of MR elastomers, Journal of Intelligent Material Systems and Structures, Vol. 00-2011.

[5] X. B. Nguyen, T. Komatsuzaki, Y. Iwata, H. Asanuma, Fuzzy Semiactive Vibration Control of Structures Using Magnetorheological Elastomer, Shock and Vibration, Vol. 2017, Article ID 3651057, 15 pages.

[6] R. Li, L. Z. Sun, Dynamic viscoelastic modeling of magnetorheological elastomers, Acta Mech, 225 (2014), pp. 1347-1359.

[7] W. H. Li, Y. Zhou, T. F. Tian, Viscoelastic of MR elastomers under harmonic loading, Rheol Acta, 49 (2010), pp. 733-740.

[8] S. H. Eem, H. J. Jung, Jung, J. H. Jung, Modeling of magneto-rheological elastomers for harmonic shear deformation, IEEE transactions on magnetics, 48 (11) (2012), pp. 3080-3083.

[9] J. Yang, H. Du, W. Li, Y. Li, J. Li, S. Sun, H. X. Deng, Experimental study and modeling of a novel magnetorheological elastomer isolator, Smart Materials and Structures, 22 (11) (2013), pp. 1-14. 
[10] M. Norouzi, S. M. S. Alehashem, H. Vatandoost, M. M. Shahmardan, A new approach for modeling of magnetorheological elastomers, Journal of Intelligent Material Systems and Structures, 27(8) (2016), pp. 1121-1135.

[11] G. J. Liao, X. L. Gong, C. J. Kang, S. H. Xuan, The design of an active-adaptive tuned vibration absorber based on magnetorheological elastomer and its vibration attenuation performance, Smart Material and Structures, 20(07) (2011), pp. 5015-5025.

[12] W. Zhu, X.-t. Rui, Semiactive Vibration Control Using a Magnetorheological Damper and a Magnetorheological Elastomer Based on the Bouc-Wen Model, Shock and Vibration, Vol. 2014, Article ID 405421, 10 pages.

[13] S. Opie, W. Yim, Design and control of a real-time variable modulus vibration isolator, Journal of Intelligent Material Systems and Structures, 22(2) (2010), pp. 113-125.

[14] M. Behrooz, X. Wang, F. Gordaninejad, Performance of a new magnetorheological elastomer isolator system, Smart Material and Structures, 23(04) (2013), 5014(8pp).

[15] H. Du, W. Li, N. Zhang, Semi-active variable stiffness vibration control of vehicle seat suspension using an MR elastomer isolator, Smart Material and Structures, 20(10) (2011), 5003 (10pp).

[16] K. M. Choi, S. W. Cho, H. J. Jung and I. W. Lee, Semi-active fuzzy control for seismic response reduction using magnetorheological dampers, Earthquake Engineering and Structural Dynamics, 2004; 33:723-736.

[17] M. Berg, A non-linear rubber spring model for rail vehicle dynamics analysis, Vehicle System Dynamics, 30 (1998), pp. 197-212.

[18] R.S. Mills, Model Test on Earthquake Simulators: Development and Implementation of Experimental Procedures, 1979. 